\title{
Photodoping and Fast Charge Extraction in Ionic Carbon Nitride Photoanodes
}

Christiane Adler, ${ }^{\dagger[a]}$ Shababa Selim, ${ }^{+[b]}$ Igor Krivtsov, ${ }^{[a]}$ Chunyu Li, ${ }^{[c, d]}$ Dariusz Mitoraj, ${ }^{[a]}$ Benjamin Dietzek, ${ }^{[c, d, e]}$ James R. Durrant, ${ }^{*[b]}$ Radim Beranek ${ }^{*[a]}$

Department of Chemistry and Centre for Processable Electronics, MSRH, White City Campus, Imperial College London, London W12 0BZ, United Kingdom

c Department Functional Interfaces, Leibniz Institute of Photonic Technology Jena (IPHT), AlbertEinstein-Straße 9, 07745 Jena, Germany Institute of Physical Chemistry, Friedrich-Schiller University Jena, Helmholtzweg 4, 07743 Jena, Germany

e Centre for Energy and Environmental Chemistry Jena (CEEC Jena), Friedrich-Schiller University Jena, Philosophenweg 7a, 07743 Jena, Germany

$+\quad$ Authors contributed equally.

* Corresponding authors: Radim Beranek (radim.beranek@uni-ulm.de) James R. Durrant (j.durrant@imperial.ac.uk) 


\begin{abstract}
Ionic carbon nitrides based on poly(heptazine imides) $(\mathrm{PHI})$ represent one of the most vigorously studied class of low-cost, tunable and stable polymeric materials with possible applications in photocatalysis and energy storage. However, the fundamental photophysical properties and processes that govern the performance of these materials are still poorly understood and have been studied mostly in form of particle suspensions. We study, for the first time, the photogenerated charge dynamics in highly stable and binder-free $\mathrm{PHI}$ photoanodes with excellent performance in photoelectrocatalytic alcohol conversions using in operando transient photocurrents (TPC) and spectroelectrochemical photoinduced absorption (PIA) measurements. Interestingly, we discover that light-induced accumulation of long-lived trapped electrons within the $\mathrm{PHI}$ film leads to effective photodoping of the $\mathrm{PHI}$ film, resulting in a significant improvement of photocurrent response due to more efficient electron transport. While photodoping has been previously reported for various inorganic and organic semiconductors, the here reported beneficial photodoping effect has never been shown before for carbon nitride materials. Furthermore, we find that the extraction kinetics of untrapped electrons are remarkably fast in these PHI photoanodes, with electron extraction times (ms) comparable to those measured for commonly employed metal oxide semiconductors (e.g., $\mathrm{TiO}_{2}, \mathrm{WO}_{3}, \mathrm{BiVO}_{4}$ ). These results shed light on the excellent performance of $\mathrm{PHI}$ photoanodes in alcohol photoreforming, including very negative photocurrent onset, outstanding fill factor, and the possibility to carry out photoreforming under zerobias conditions. More generally, the here reported photodoping effect and fast electron extraction in PHI photoanodes represent thus yet another intriguing property of ionic (PHI-based) carbon nitride materials, and establish a strong rationale for the use of $\mathrm{PHI}$ films in various applications, such as bias-free photoelectrochemistry or photobatteries.
\end{abstract}




\section{Introduction}

Polymeric carbon nitride (PCN) materials represent one of the most vigorously studied class of low-cost, tunable and highly stable polymeric photocatalysts, with potential applications in light-driven hydrogen production, selective oxidations of organic substrates and other useful chemical transformations. ${ }^{[1]}$ The fundamental photophysical properties and processes (photoexcitation, recombination, charge separation, photoluminescence) that govern the performance of PCN-based photocatalysts have been intensely studied, ${ }^{[2]}$ revealing many aspects related to the electron transfer within and between different $\mathrm{PCN}$ units, ${ }^{[2]]}$ charge separation ${ }^{[2 a, b]}$ and recombination ${ }^{[2]}$ processes, in addition to providing insight into the hole transfer to electron donors. ${ }^{[2 e]}$

Recently, there has been increasing interest in poly(heptazine imide) (PHI)-based materials, a class of (semi)crystalline ionic PCNs comprising stacks of two-dimensional heptazine-based networks and alkali metal cations and/or protons. ${ }^{[1 a, 3]}$ Compared to conventional PCN materials, the PHI photocatalysts often exhibited higher photocatalytic activity owing to their surface functional (e.g., cyanamide) groups and thus improved dispersibility. ${ }^{[1 a, 3-4]}$ Moreover, it has been demonstrated that the photogenerated electrons trapped within $\mathrm{PHI}$ materials can be effectively accumulated and stored, ${ }^{[2 h, 5]}$ in order to be later utilized, for example, for hydrogen evolution in the dark upon addition of a co-catalyst, ${ }^{[6]}$ or in solar batteries. ${ }^{[5 a]}$ While some studies have suggested that structural defects (e.g., cyanamide moieties) can be beneficial for photocatalytic activity, $\left.{ }^{[2,}, \mathrm{i}, 4 \mathrm{~b}, 6 \mathrm{6c}\right]$ the presence of deep electron traps and resulting electron accumulation and faster recombination have been typically identified as the principal bottleneck in efficient operation of both PHI-based and conventional PCN photocatalysts. ${ }^{[2 c, d, g, ~ h] ~}$

Most research to date on the fundamental photophysics of PCN-based materials has been carried out using powder suspensions, since the fabrication of stable PCN-based photoelectrodes is very challenging, mainly due to the poor adhesion of PCN to conductive substrates ${ }^{[5 a, 7]}$ and the poor conductivity of PCN films, hindering efficient charge extraction into the external circuit. ${ }^{[8]}$ Therefore, the very scarce spectroscopic studies of conventional PCN photoelectrodes have been typically carried out using binders such as Nafion or $\mathrm{TiO}_{2}$ electron-transport layers, ${ }^{[2 a, 9]}$ which can complicate understanding of the observed behaviour. To the best of our knowledge, there are no reports available on fundamental photophysical properties of photoelectrodes based on ionic carbon nitrides (PHI).

We have recently reported a novel method for fabrication of thin, binder-free $(\sim 400-500 \mathrm{~nm}) \mathrm{PHI}$ photoanodes exhibiting excellent long-term mechanical and photoelectrochemical stability. ${ }^{[10]}$ The synthetic process capitalizes on the use of small $(\sim 10 \mathrm{~nm})$ nanoparticles of alkali metal-containing poly(heptazine imide) $(\mathrm{K}, \mathrm{Na}-\mathrm{PHI}){ }^{[4 a]}$ which are fully soluble in water and enable an easy sol-gel processing, yielding highly stable $\mathrm{PHI}$ films on various substrates including FTO.[10] Our PHI photoanodes exhibited selective ( $\sim 100 \%$ Faradaic efficiency) photoelectrocatalytic conversion of various alcohols, standing out, in particular, by their very low photocurrent onset potential and exceptionally high fill factor, thus permitting effective photoreforming of alcohols to hydrogen even without any applied electric bias. ${ }^{[10]}$

Herein, we provide, for the first time, in operando investigations of photoelectrochemical processes in the highly stable, binder-free $\mathrm{PHI}$ photoanodes. We employ transient photocurrent (TPC) and 
spectroelectrochemical photo-induced absorption spectroscopy (PIAS) measurements that enable us to directly observe photogenerated charge dynamics under operando conditions and correlate these with the concomitantly measured photocurrent. This allows us to observe the impact of charge accumulation within our PHI photoanodes on photocurrent generation from methanol oxidation. We have previously employed these techniques to study photoelectrochemical processes in various metal oxide photoelectrodes. ${ }^{[11]}$ Interestingly, we find that the excellent performance of the PHI photoanodes originates, at least in part, from fast electron extraction on a timescale similar to that observed for commonly employed inorganic photoanodes $\left(\mathrm{TiO}_{2}, \mathrm{WO}_{3}, \mathrm{BiVO}_{4}\right)$. Furthermore, our findings suggest that extensive electron trapping and accumulation results in effective photodoping in $\mathrm{PHI}$ photoanodes, which leads to enhanced electron transport, improving thus the photoelectrocatalytic activity. While photodoping effects upon light-induced electron accumulation have been previously reported for various inorganic ${ }^{[12]}$ and organic semiconductors, ${ }^{[13]}$ we demonstrate for the first time that photodoping can be operative also in photoelectrodes based on ionic carbon nitrides.

\section{Results and Discussion}

The structural, optical and photoelectrochemical properties of our porous $\mathrm{PHI}$ photoanodes (PHI$\mathrm{DB} / \mathrm{S} 450$ ) were reported in detail in Ref. ${ }^{100}$. While the PHI photoanodes did not oxidize water, the photocurrents during oxidation of alcohols, such as methanol, were among the highest observed for PCN-based photoanodes. This is exemplified in Figure 1a, which also shows the exceptionally high fill factor of the J-V curve, across a wide potential range. This feature is reminiscent of the behaviour of mesoporous inorganic photoanodes, such as $\mathrm{TiO}_{2} \cdot{ }^{[14]}$ In addition, the $\mathrm{PHI}$ photoanodes outperform all PCN-based photoanodes and even most inorganic semiconductors in terms of the very negative photocurrent onset potential (below $0 \mathrm{~V}$ vs. RHE). This allows for effective photoreforming of methanol to hydrogen even under bias-free conditions (Figure $1 \mathrm{~b}$ ), which is - to the best of our knowledge unprecedented behaviour for photoanodes based on organic polymers. In addition, the absence of the photocurrent spikes in the linear sweep voltammogram (LSV) of the PHI-DB/S450 film even at potentials down to $0 \mathrm{~V}$ vs. RHE indicates the absence of surface recombination losses at the PHI-electrolyte interface (Figure 1a). ${ }^{[11 a, 15]}$

Photocurrent transients measured for the PHI photoanodes when illuminated by a LED pulse for 5 seconds ( $365 \mathrm{~nm}$ LED, $5 \mathrm{~mW} \mathrm{~cm}^{-2}$ ) allow us to study the effect of the pre-illumination on the observed photocurrents for methanol oxidation, as shown in Figure 1c. We do this by pre-illuminating the sample under applied potential (1.1 V vs. RHE) with continuous LED irradiation (also $365 \mathrm{~nm}, 5 \mathrm{~mW} \mathrm{~cm}^{-2}$ ) in the presence of methanol, prior to measuring the photocurrent transient with the LED pulse. From Figure 1c, we observe that pre-illumination not only leads to a direct enhancement in the obtained photocurrents, but also affects the speed of saturation of the photocurrent. Photocurrent saturation (plateau) implies that the system has reached steady-state conditions, whereby the surface concentration of photogenerated holes is not changing and the flux of photogenerated holes into the surface is balanced by the flux of holes across the interface contributing to the photocurrent (i.e., rate of methanol oxidation) and by the flux of holes due to (surface or sub-surface) recombination. We point out that the so-called photocurrent doubling effect can be ruled out in our case since the half-wave 
oxidation potential of hydroxymethyl radical (the product of one-electron oxidation of methanol) is ca. $0.32 \mathrm{~V}$ vs. RHE, which makes the injection of an electron from the radical to the conduction band edge of PHI-DB/S450 (-0.63 V vs. RHE) rather unlikely. ${ }^{[10]}$ Our pulsed LED induced photocurrent transients (Figure 1c) suggest that a pre-illumination time of $\sim 10 \mathrm{~min}$ is sufficient for the system to reach optimum performance. This was further confirmed from measurement of the photocurrents during pre-illumination using chronoamperometry (Figure S1), where we can observe that the photocurrent continues to increase and plateaus within a period of 7-10 mins of pre-illumination. Intriguingly, the slow rise of the photocurrent transient without pre-illumination (Figure 1c, black trace), most likely related to the typically poor intrinsic conductivity of PCN materials, accelerates significantly with prolonged pre-illumination of the system (Figure 1c, blue trace). This behaviour has some similarity to photodoping effects reported previously for various inorganic ${ }^{[12]}$ and organic ${ }^{[13]}$ semiconductors, as we discuss further later.
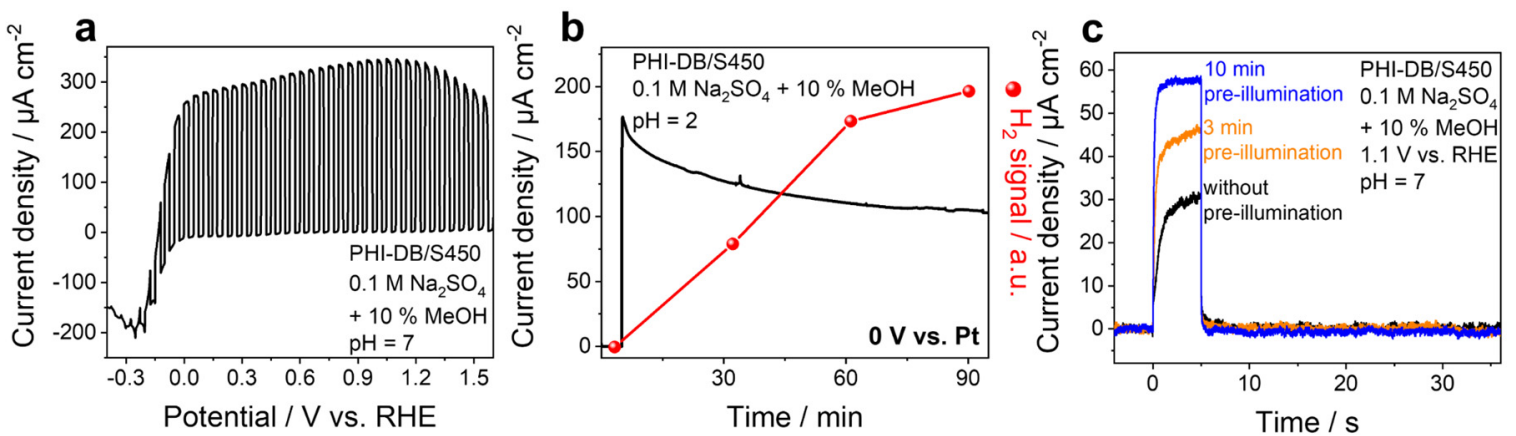

Figure 1. Photoelectrochemical characteristics of PHI carbon nitride photoanodes for methanol oxidation. a) LSV curves (scanned in cathodic direction with a sweep-rate of $5 \mathrm{mV} \mathrm{s}^{-1}$ ) of PHI-DB/S450 in $0.1 \mathrm{M} \mathrm{Na}_{2} \mathrm{SO}_{4}$ with $10 \mathrm{v} \%$ methanol ( $\mathrm{pH} 7.0$ ) upon on/off illumination (2 sun, $5 \mathrm{~s}$ light/ $5 \mathrm{~s}$ dark) from back side. b) Bias-free photoreforming of methanol: photocurrent (black) and $\mathrm{H}_{2}$ evolution (red) detected by the GC-BID for PHI-DB/S450 in a two-electrode setup ( $0 \mathrm{~V}$ vs. Pt counter electrode; 1 sun illumination; $0.1 \mathrm{M} \mathrm{Na}_{2} \mathrm{SO}_{4}$ with $10 \% \mathrm{v} / \mathrm{v}$ methanol; for this experiment the $\mathrm{pH}$ was adjusted to $\mathrm{pH} 2$ in order to decrease the ohmic drop in the electrolyte). c) Photocurrent transients measured of PHI-DB/S450 in $0.1 \mathrm{M} \mathrm{Na}_{2} \mathrm{SO}_{4}+10 \mathrm{v} \%$ methanol at $1.1 \mathrm{~V}$ vs. RHE without (black), with 3 min (amber) or $10 \mathrm{~min}$ (blue) pre-illumination directly before measurement. Excitation with $365 \mathrm{~nm}$ LED $\left(5 \mathrm{~mW} \mathrm{~cm}^{-}\right.$ $\left.{ }^{2}\right)$ for $5 \mathrm{~s}$ and dark for $45 \mathrm{~s}$.

Next, we employ photoinduced absorption (PIA) spectroscopy as an operando probe of accumulation of charges in our PHI photoanodes under the same pulsed LED irradiation employed for the photocurrent transients shown in Figure 1c, with/without pre-illumination. The PIA spectra of our PHI photoanodes shown in Figure 2a present a relatively broad spectral feature across the visible region probed herein $(460 \mathrm{~nm}-800 \mathrm{~nm}$ ), with a maximum at $\sim 600 \mathrm{~nm}$ when recorded without any preillumination (black trace). No change in the magnitude of the optical signals is observed when illuminating with a higher light intensity LED pulse (green trace, $10 \mathrm{~mW} \mathrm{~cm}^{-2}$ ). The differential optical density signal diminishes significantly upon pre-illumination (blue trace) leaving a flat featureless spectrum across the probed region. Furthermore, the loss of the optical signal in the absence of methanol (red trace), which effectively scavenges the photogenerated holes at the surface, ${ }^{[2 e]}$ indicates that the observed signals are likely to be related to photogenerated electrons.

Figure $2 \mathrm{~b}$ plots the kinetics of the PIA signal at $650 \mathrm{~nm}$ induced by pulsed LED irradiation without preillumination, with/without methanol. When the LED pulse is turned off, the PIA signal decays very slowly in the presence of methanol ( $\left.\mathrm{t}_{50 \%} \sim 20-30 \mathrm{~s}\right)$. A faster decay of the PIA signal is observed in the absence 
of methanol, attributed to the absence of methanol resulting in unreacted photogenerated holes recombining with accumulated electrons, and further supporting our assignment of the PIA signal to electrons. In contrast to these slow PIA decays, the concomitant transient photocurrent measurements (Figure 1c) show a rapid drop in the photocurrent to zero when the LED is switched off. The slow nature of the optical signal decay, consistent with previous reports of carbon nitrides, ${ }^{[2 c, ~ d, ~ h, ~ 5 a, ~ 6 c] ~ i n d i c a t e s ~ t h i s ~}$ signal originates primarily from relatively immobile and therefore deeply trapped, electrons. Furthermore, consistent with the J-V characteristics (Figure 1a), the magnitude of the optical signal and the photocurrents in the absence of pre-illumination are observed to be largely independent of the applied bias (Figure S2). This is in agreement with the very good fill factor of our PHI films indicating that the applied potential has only a minor effect on the charge accumulation and extraction properties of the photoanode (Figure 1a).

\section{a}

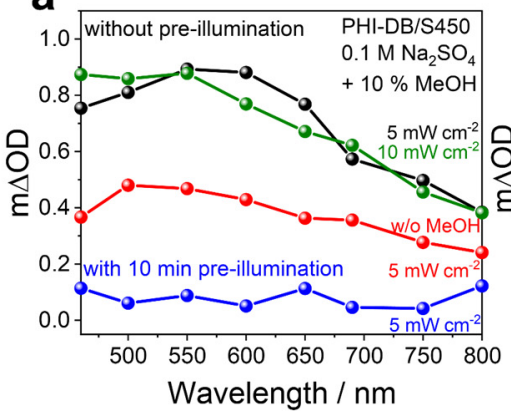

b

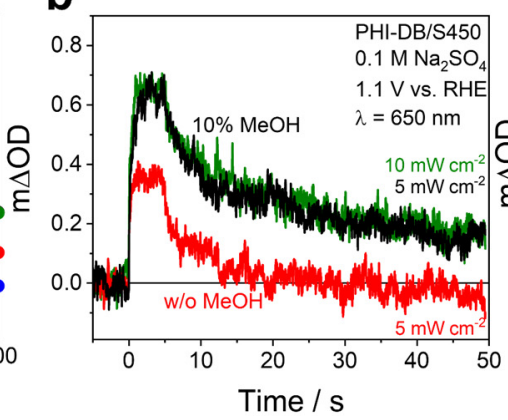

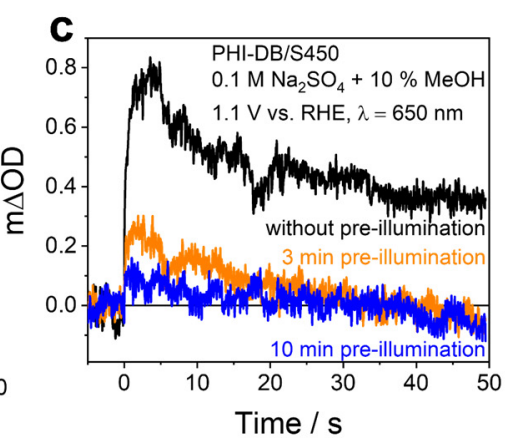

Figure 2. Spectroelectrochemical characteristics of PHI carbon nitride photoanodes with and without preillumination. a) Comparison of PIA spectra with $365 \mathrm{~nm}$ LED excitation $\left(5 \mathrm{~mW} \mathrm{~cm}^{-2}\right.$ (black) and $10 \mathrm{~mW} \mathrm{~cm}^{-}$ ${ }^{2}$ (green)) without any pre-illumination, and with a 10 min pre-illumination (blue), measured in $0.1 \mathrm{M} \mathrm{Na}_{2} \mathrm{SO}_{4}$ with 10 $\mathrm{v} \% \mathrm{MeOH}$. Spectrum obtained without pre-illumination in absence of any $\mathrm{MeOH}$ is also shown (red). b) PIA optical trace and decay monitored at $\lambda=650 \mathrm{~nm}$ for PHI-DB/S450 photoanodes in absence of any pre-illumination, when excited with $365 \mathrm{~nm}$ LED $\left(5 \mathrm{mw} \mathrm{cm}^{-2}\right.$ (black) and $10 \mathrm{~mW} \mathrm{~cm}^{-2}$ (green) in $0.1 \mathrm{M} \mathrm{Na}_{2} \mathrm{SO}_{4}$, with $10 \mathrm{v} \% \mathrm{MeOH}$. Analogous measurement in absence of any $\mathrm{MeOH}$ is also shown (red). The samples were excited with a LED pulse for $5 \mathrm{~s}$ and subsequently left in the dark for $45 \mathrm{~s}$ during the measurement. c) PIA of PHI-DB/S450 in $0.1 \mathrm{M} \mathrm{Na}_{2} \mathrm{SO}_{4}$ $+10 \mathrm{v} \% \mathrm{MeOH}$ without (black), with $3 \mathrm{~min}$ (amber) or $10 \mathrm{~min}$ (blue) pre-illumination directly before measurement at $650 \mathrm{~nm}$. Excitation with $365 \mathrm{~nm} \mathrm{LED}\left(5 \mathrm{~mW} \mathrm{~cm}^{-2}\right)$ for $5 \mathrm{~s}$ and left in dark for $45 \mathrm{~s}$. All measurements were obtained at $1.1 \mathrm{~V}$ vs. RHE.

We now employ PIA to investigate the effect of pre-illumination on the accumulation of photogenerated electrons, shown in Figure 2c. Upon pre-illumination, there is a considerable loss in the magnitude of PIA signal at $650 \mathrm{~nm}$, combined with an acceleration of its decay kinetics. In addition, as shown in Figure S3, increasing the light intensity from $5 \mathrm{~mW} \mathrm{~cm}^{-2}$ to $10 \mathrm{~mW} \mathrm{~cm}^{-2}$ for both the pre-illumination steps and the subsequent PIA / photocurrent transients results in the following effects: (i) an increase in the photocurrent density, (ii) earlier stabilisation of the photocurrent density within 3 mins of pre-illumination (vs. $\sim 10$ mins for $5 \mathrm{~mW} \mathrm{~cm}^{-2}$ ), correlated with a (iii) concomitant earlier decrease in the optical signal (by 3 mins). Critically, the magnitude of the PIA signal without pre-illumination (assigned above to photoinduced trapped electrons) is also found to be unaffected by the light intensity (Figure $2 \mathrm{~b}$ and Figure S3). We propose that this behaviour is related to the gradual filling of trap states by photogenerated electrons during illumination, resulting in significant electron accumulation within the film. Further, the data also indicates the requirement for a critical threshold of electron accumulation that leads to optimal performance in this system. We assume that the observed electron accumulation corresponds to the formation of $\mathrm{PHI}^{\circ-}$ anion radicals known to occur in ionic $\mathrm{PHI}$-type materials. ${ }^{[2 \mathrm{c}, \mathrm{h}, 3 \mathrm{~b} \text {, }}$ 
5, 6c] These accumulated electrons are only slowly scavenged by oxygen in the dark, ${ }^{[2 h]}$ and exhibit long carrier lifetimes (> $30 \mathrm{~s}$, Figure $2 \mathrm{~b}, \mathrm{c}$ ) when surface holes have been scavenged by methanol, highlighting the unreactive nature of these trapped charges (Figure 2a, b). On the other hand, it was previously reported that accumulated electrons in ionic PCNs can be utilized for hydrogen evolution in the presence of a co-catalyst. ${ }^{[2 g, 6]}$ The fact that electron trapping and accumulation in our PHI photoanodes does not impair but rather improves their photoelectrocatalytic performance is rather intriguing, especially in view of previous reports on the detrimental effects of electron trapping on the performance of PCN-based photocatalysts. ${ }^{[2 c,}$ d, g] For example, our previous spectroscopic investigations of ionic-type PCN in form of particle suspensions have demonstrated accelerated recombination upon accumulation of long-lived, trapped electrons. ${ }^{[2]}$ In order to investigate this further, we applied nanosecond-transient absorption (ns-TA) spectroscopy under bias-free conditions to investigate the charge kinetics of the PHI-DB/S450 films on a timescale of 50 ns to $5 \mu$ s in the presence of air, water and methanol (see Note $\mathbf{S 1}$ in the Supporting Information). We observe a faster decay of the optical signal in the presence of methanol, consistent with enhanced recombination resulting from more electrons accumulation in the material in the presence of effective hole scavenger (Figure S4), in line with our previous results. ${ }^{[2 c]}$ However, such negative effects of electron accumulation in our PHI$\mathrm{DB} / \mathrm{S} 450$ photoanodes are clearly outbalanced by the positive effect of trap passivation, as evidenced by enhanced photocurrent generation (Figure 1c) which is superior to that typically encountered in conventional (non-ionic) PCN materials. In this context, we speculate that, due to the distinct nature of the ionic PHI-based materials and the highly porous nature of our PHI-DB/S450 photoanodes ${ }^{[10]}$ (as compared to a dense analogue that shows much lower photocurrents, Figure S5), the negative charge of the accumulated trapped electrons in the PHI photoanodes can be effectively screened by cations $\left(\mathrm{K}^{+}, \mathrm{Na}^{+}, \mathrm{H}^{+}\right)$from the film and from the solution, which may at least partially eliminate the detrimental effects of charge accumulation. We find that electron accumulation during pre-illumination is reversible, shown in Figure S6. The loss in the optical signal (i.e., due to electron accumulation) resulting from repeated pre-illumination cycles (blue trace) is recovered to the initial optical trace observed prior to any pre-illumination when the sample has been left in the dark over a period of 24 hours (black trace). This reversible behaviour demonstrates that our photoanodes are not suffering oxidation or degradation. Rather, the loss in the optical signal and the concomitant increase in the photocurrent is attributed to the filling of trap states with long-lived unreactive charge carriers.

Our results indicate that long lived electron accumulation in our PHI photoanodes is correlated with a significant increase of the overall photocurrent, assigned to trap filling. This beneficial effect of electron accumulation can be understood as resulting from an effective photodoping of the PHI film, an effect that has been observed for a range of inorganic ${ }^{[12]}$ and organic ${ }^{[13]}$ semiconductors, however not reported previously for PCN materials. The photodoping during pre-illumination effectively fills/passivates deep electron traps and improves the charge transport through the film, as is also indicated by significantly lower impedance of the electrode upon illumination (Figure S7). In relatively disordered organic materials like our PHI films, the electron transport mechanism can be typically understood in terms of a thermally activated hopping of electrons that are relatively localized in shallow trap states. ${ }^{[16]}$ Upon effective filling of deep trap states during pre-illumination (i.e., by photodoping), the electron trapping is 
avoided and the electron transport through the PHI film is effectively enhanced (Figure S8), as we investigate in more detail below.
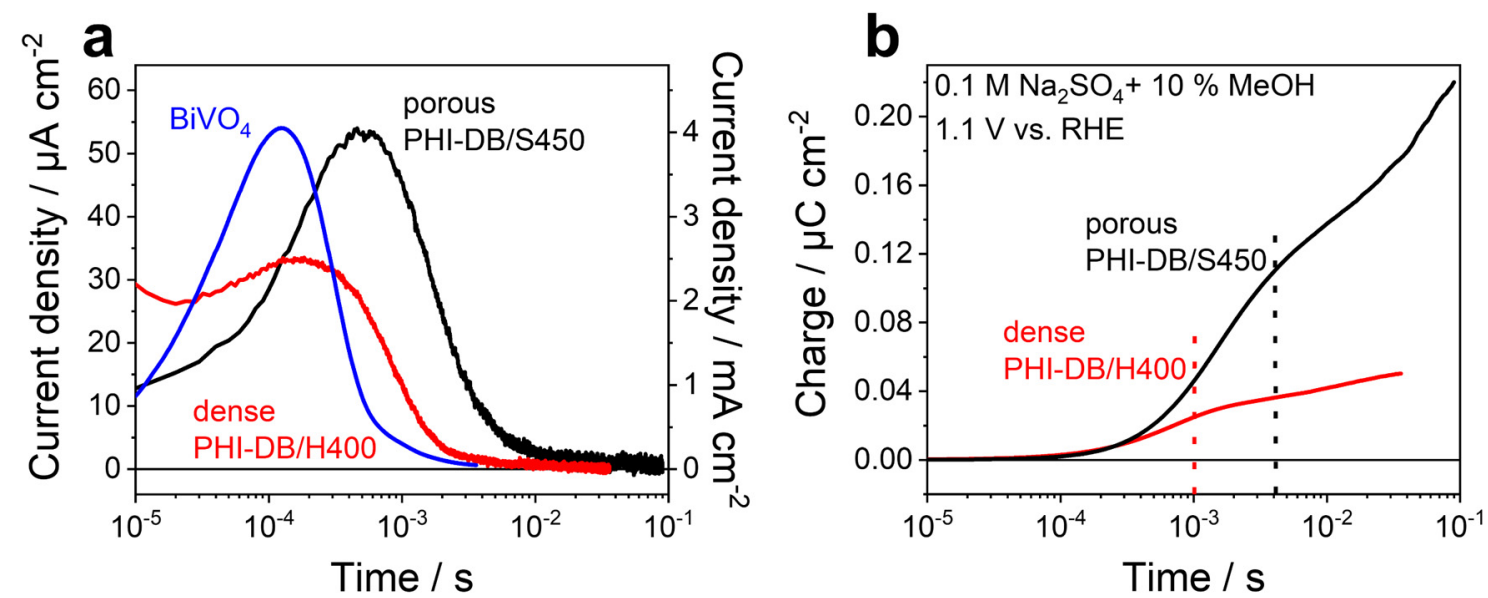

Figure 3. Charge extraction characteristics of PHI carbon nitride photoanodes. a) Transient photocurrents (TPC) and b) integrated TPCs showing the total charges extracted from porous PHI-DB/S450 (black) and dense $\mathrm{PHI}-\mathrm{DB} / \mathrm{H} 400$ (red) photoanodes in $0.1 \mathrm{M} \mathrm{Na}_{2} \mathrm{SO}_{4}+10 \mathrm{v} \% \mathrm{MeOH}$. The samples were excited from the back with a $355 \mathrm{~nm}$ pulsed laser at $1.1 \mathrm{~V}$ vs. RHE. For comparison with typical metal oxides, TPC (right axis) for a dense $\mathrm{BiVO}_{4}$ photoelectrode (of thickness $400-250 \mathrm{~nm}$ ) in $0.1 \mathrm{M} \mathrm{KP}$ buffer for front side excitation with a $355 \mathrm{~nm}$ pulsed laser at $1.23 \mathrm{~V}$ vs. RHE is shown in a.

We now turn to the electron extraction kinetics of our $\mathrm{PHI}$ photoanodes using transient photocurrent (TPC) measurements employing short pulse (ns) laser photoexcitation (Figure 3). We compare the charge extraction transients of our porous $\mathrm{PHI}$ photoanode (PHI-DB/S450) with a dense (i.e., nonporous) analogue (PHI-DB/H400). ${ }^{[10]}$ The dense film, although having similar thicknesses ( 400-500 $\mathrm{nm}$ ) and nearly the same intrinsic structural and physicochemical properties as PHI-DB/S450, has much lower photoelectrocatalytic activity and did not show any notable effect of pre-illumination (Figure S5), attributed to its lower surface area and correspondingly lower ability to accumulate charges. ${ }^{[10]}$ The timescale of the TPC maxima (Figure 3a), which represents the highest rate of electron extraction, are $\sim 0.4 \mathrm{~ms}$ for porous (PHI-DB/S450) and $\sim 0.2 \mathrm{~ms}$ for dense (PHI-DB/H400) photoanodes. The faster electron extraction times observed for dense compared to porous $\mathrm{PHI}$ films is consistent with the different morphologies of the samples, since the electrons in the dense film are extracted via direct path to the back contact, whereas the effective pathlength to the back contact is longer in the porous $\mathrm{PHI}$ layer. As these TPC transients employ relatively intense laser pulses $\left(300 \mu \mathrm{J} \mathrm{cm}{ }^{-2}, 0.8 \mathrm{~Hz}\right.$ repetition rate), it is likely they induce significant trap filling / photodoping. This supposition is supported by the only minor dependence of the TPC kinetics with and without background light bias (LED, $5 \mathrm{~mW} \mathrm{~cm}^{-2}$ ), shown in Figure S9. The integrated current densities obtained from these TPC measurements show a much higher yield of total charge extracted for the porous photoelectrode in comparison to the dense analogue (Figure $3 \mathrm{~b}$ ), consistent with the corresponding steady-state J-V performance (Figure S5). This is attributed to more efficient methanol oxidation by photogenerated holes due to the higher active surface area of the porous film. The half-time for charge extraction ( $\mathrm{t}_{50 \%}$ rise time in Figure $3 \mathrm{~b}$ ) for the dense (PHI-DB/H400) film is $1 \mathrm{~ms}$, which is faster than $4 \mathrm{~ms}$ observed for the porous one (dot line, Figure $3 b$ ), attributed to the differences in film morphology discussed above. Interestingly, the charge 
extraction time is very similar for back or front side irradiation for both samples (Figure S11), which can be explained by the large optical penetration depth of the laser beam (ca. 250 and $350 \mathrm{~nm}$ for stand for $\mathrm{PHI}-\mathrm{DB} / \mathrm{S} 450$ and $\mathrm{PHI}-\mathrm{DB} / \mathrm{H} 400$, respectively $)^{[10]}$ and the relatively long electron diffusion length of ca. $430 \mathrm{~nm}$ (i.e., in the range of the thickness of the sample) ${ }^{[10]}$ determined from modelling the external quantum efficiency data obtained without pre-illumination, i.e., before a complete photodoping is reached (Figure S12). Remarkably, the timescale (ms) of electron extraction for our PHI photoelectrodes is on the same order of magnitude as bismuth vanadate $\left(\mathrm{BiVO}_{4}\right)$ photoanodes of comparable thickness, ${ }^{[17]}$ one of the best-performing metal oxide photoanode materials for water oxidation to date.

A schematic representation of electron dynamics within the porous PHI film is shown in Scheme 1. From the data herein, it is apparent that the PHI films exhibit significant trapping of photogenerated electrons in deep trap states, giving rise to long-lived ( $30 \mathrm{~s}$ ) trapped electrons. Under prolonged (> $1 \mathrm{~s}$ ) irradiation, these trap states become filled with electrons. This results in passivation of these trap states, effectively photodoping the material and improving the efficiency of electron extraction to the external circuit and enhancing photoelectrochemical performance. This photodoping at least partially mitigates the negative impact of the presence of deep trap states in these photoanodes. Remarkably fast (ms) electron extraction kinetics are observed for such a disordered material, assigned to electrons which avoid deep trapping, and consistent with the excellent fill factor of the PHI photoanodes (Figure 1a). On the other hand, without any effective hole scavenger, such as the methanol employed herein, recombination of trapped electrons and VB holes occurs on the seconds timescale (see Figure 2b, red trace), preventing significant water oxidation and resulting in negligible photocurrent densities in the absence of methanol (Figure S13). Enhancing the kinetics and efficiency of water oxidation by, for example, deposition of co-catalysts ${ }^{[18]}$ would be essential to extend the use of $\mathrm{PHI}$ photoanodes from photoelectrocatalytic alcohol conversions to overall water splitting.

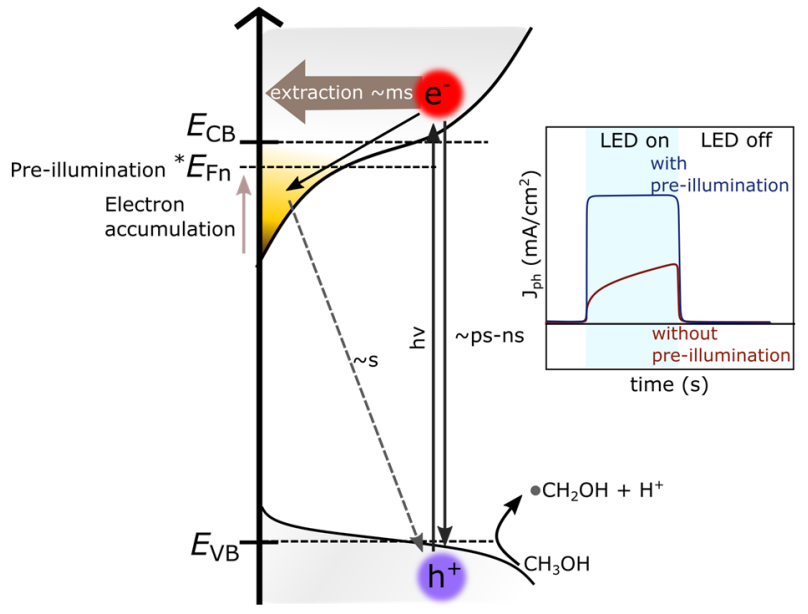

Scheme 1. Schematic illustration of processes governing the photocurrent generation in porous PHI films. The accumulation of long-lived ( $>30 \mathrm{~s}$ ) electrons in trap states occurs within s-mins and leads to effective photodoping of the PHI film, which enables very fast $(\sim 0.4 \mathrm{~ms})$ electron extraction from the PHI film into the FTO electrode and improves significantly the photocurrent response. $E_{\mathrm{vB}}, E_{\mathrm{CB}}$ and ${ }^{*} E_{\mathrm{Fn}}$ stand for the valence band edge, the conduction band edge, and the quasi-Fermi level of electrons under illumination, respectively. 


\section{Conclusion}

We have studied, for the first time, the photogenerated charge dynamics in stable and binder-free ionic (PHI) carbon nitride photoanodes using transient photocurrents (TPC) and in operando spectroelectrochemical photoinduced absorption (PIA) measurements. Consistent with previous reports, ${ }^{[2 c, d, g, h]}$ we observe prolonged irradiation results in electron accumulation in long-lived trap states within the PHI film during illumination. While it is well-established that the electrons trapped in $\mathrm{PHI}$ can be utilized for subsequent redox transformations, ${ }^{[2 h, 5]}$ we demonstrate, for the first time, that the electrons accumulated in the $\mathrm{PHI}$ film during illumination passivate deep trap states and thus also effectively photodope the PHI film. This photodoping effect leads to a significant improvement of photocurrent response due to more efficient electron transport. Recombination of trapped electrons with photogenerated holes is suppressed when the hole transfer reaction is fast (e.g., in methanol oxidation). In contrast, the slower and kinetically more challenging hole reactions (e.g., water oxidation) are outcompeted by recombination. While photodoping has been previously reported for various inorganic ${ }^{[12]}$ and organic materials, ${ }^{[13]}$ the here reported beneficial photodoping effect has not been shown before for polymeric carbon nitrides. Moreover, we demonstrate that electrons which avoid deep trapping in these $\mathrm{PHI}$ photoanodes exhibit fast (ms) extraction kinetics, with electron extraction times comparable to those measured for commonly employed metal oxide semiconductors (e.g., $\mathrm{TiO}_{2}, \mathrm{WO}_{3}, \mathrm{BiVO}_{4}$ ). These results shed light on the excellent performance of $\mathrm{PHI}$ photoanodes in alcohol photoreforming, including very negative photocurrent onset, outstanding fill factor, and the possibility to carry out photoreforming under zero-bias conditions.${ }^{[10]}$ More generally, the here reported photodoping effect and fast electron extraction in PHI photoanodes represent yet another intriguing property of the ionic (PHI-based) carbon nitride materials, and establish a strong rationale for the use of $\mathrm{PHI}$ films in various applications, such as biasfree photoelectrochemistry or photobatteries. ${ }^{[5 a, c]}$

Supporting Information. Experimental details; additional spectroscopic and photoelectrochemical results (Note S1; Figures S1-S13)

\section{Acknowledgements}

This work was funded by the Deutsche Forschungsgemeinschaft (DFG, German Research Foundation) - Projektnummer BE 5102/5-1 and 364549901 - TRR 234 CataLight [Project B6]. I.K. acknowledges the support of the Alexander von Humboldt Foundation through the Humboldt Research Fellowship. S.S. acknowledges funding from European Union's Horizon 2020 (SUN2CHEM, grant agreement 884444). 


\section{References}

[1] a) A. Savateev, M. Antonietti, ChemCatChem 2019, 11, 6166; b) X. Wang, K. Maeda, A. Thomas, K. Takanabe, G. Xin, J. M. Carlsson, K. Domen, M. Antonietti, Nat. Mater. 2009, 8, 76; c) X. Wang, S. Blechert, M. Antonietti, ACS Catal. 2012, 2, 1596; d) A. Savateev, M. Antonietti, ACS Catal. 2018, 8, 9790.

[2] a) C. Ye, J.-X. Li, Z.-J. Li, X.-B. Li, X.-B. Fan, L.-P. Zhang, B. Chen, C.-H. Tung, L.-Z. Wu, ACS Catal. 2015, 5, 6973; b) H. Zhang, Y. Chen, R. Lu, R. Li, A. Yu, Phys. Chem. Chem. Phys. 2016, 18, 14904; c) W. Yang, R. Godin, H. Kasap, B. Moss, Y. Dong, S. A. J. Hillman, L. Steier, E. Reisner, J. R. Durrant, J. Am. Chem. Soc. 2019, 141, 11219; d) R. Godin, Y. Wang, M. A. Zwijnenburg, J. Tang, J. R. Durrant, J. Am. Chem. Soc. 2017, 139, 5216; e) Z. Chen, Q. Zhang, Y. Luo, Angew. Chem. Int. Ed. 2018, 57, 5320; f) K. L. Corp, C. W. Schlenker, J. Am. Chem. Soc. 2017, 139, 7904; g) H. Kasap, C. A. Caputo, B. C. Martindale, R. Godin, V. W. Lau, B. V. Lotsch, J. R. Durrant, E. Reisner, J. Am. Chem. Soc. 2016, 138, 9183; h) C. Li, E. Hofmeister, I. Krivtsov, D. Mitoraj, C. Adler, R. Beranek, B. Dietzek, ChemSusChem 2021, 14, 1728; i) A. J. Rieth, Y. Qin, B. C. M. Martindale, D. G. Nocera, J. Am. Chem. Soc. 2021, 143, 4646.

[3] a) H. Schlomberg, J. Kroger, G. Savasci, M. W. Terban, S. Bette, I. Moudrakovski, V. Duppel, F. Podjaski, R. Siegel, J. Senker, R. E. Dinnebier, C. Ochsenfeld, B. V. Lotsch, Chem. Mater. 2019, 31, 7478; b) A. Savateev, B. Kurpil, A. Mishchenko, G. Zhang, M. Antonietti, Chem. Sci. 2018, 9, 3584.

[4] a) I. Krivtsov, D. Mitoraj, C. Adler, M. Ilkaeva, M. Sardo, L. Mafra, C. Neumann, A. Turchanin, C. Li, B. Dietzek, R. Leiter, J. Biskupek, U. Kaiser, C. Im, B. Kirchhoff, T. Jacob, R. Beranek, Angew. Chem. Int. Ed. 2020, 59, 487; b) V. W. Lau, I. Moudrakovski, T. Botari, S. Weinberger, M. B. Mesch, V. Duppel, J. Senker, V. Blum, B. V. Lotsch, Nat. Commun. 2016, 7, 12165.

[5] a) F. Podjaski, J. Kroger, B. V. Lotsch, Adv. Mater. 2018, 30, 1705477; b) Y. Markushyna, P. Lamagni, C. Teutloff, J. Catalano, N. Lock, G. Zhang, M. Antonietti, A. Savateev, J. Mater. Chem. A 2019, 7, 24771; c) F. Podjaski, B. V. Lotsch, Adv. Energy Mater. 2021, 11, 2003049.

[6] a) H. Kasap, R. Godin, C. Jeay-Bizot, D. S. Achilleos, X. Fang, J. R. Durrant, E. Reisner, ACS Catal. 2018, 8, 6914; b) C. D. Windle, A. Wieczorek, L. Xiong, M. Sachs, C. Bozal-Ginesta, H. Cha, J. K. Cockcroft, J. Durrant, J. Tang, Chem. Sci. 2020, 11, 8425; c) V. W. Lau, D. Klose, H. Kasap, F. Podjaski, M. C. Pignie, E. Reisner, G. Jeschke, B. V. Lotsch, Angew. Chem. Int. Ed. 2017, 56, 510 .

[7] a) H. Ou, P. Yang, L. Lin, M. Anpo, X. Wang, Angew. Chem. Int. Ed. 2017, 56, 10905; b) J. Zhang, M. Zhang, L. Lin, X. Wang, Angew. Chem. Int. Ed. 2015, 54, 6297; c) J. Safaei, N. A. Mohamed, M. F. Mohamad Noh, M. F. Soh, N. A. Ludin, M. A. Ibrahim, W. N. Roslam Wan Isahak, M. A. Mat Teridi, J. Mater. Chem. A 2018, 6, 22346; d) J. Qin, J. Barrio, G. Peng, J. Tzadikov, L. Abisdris, M. Volokh, M. Shalom, Nat. Commun. 2020, 11, 4701; e) N. Karjule, J. Barrio, L. Xing, M. Volokh, M. Shalom, Nano Lett. 2020, 20, 4618; f) J. W. Xia, N. Karjule, L. Abisdris, M. Volokh, M. Shalom, Chem. Mater. 2020, 32, 5845.

[8] M. Shalom, S. Gimenez, F. Schipper, I. Herraiz-Cardona, J. Bisquert, M. Antonietti, Angew. Chem. Int. Ed. 2014, 53, 3654.

[9] J. Albero, E. M. Barea, J. Xu, I. Mora-Seró, H. Garcia, M. Shalom, Adv. Mater. Interfaces 2017, $4,1600265$.

[10] C. Adler, I. Krivtsov, D. Mitoraj, L. dos Santos-Gómez, S. García-Granda, C. Neumann, J. Kund, C. Kranz, B. Mizaikoff, A. Turchanin, R. Beranek, ChemSusChem 2021, 14, 2170.

[11] a) F. Le Formal, E. Pastor, S. D. Tilley, C. A. Mesa, S. R. Pendlebury, M. Gratzel, J. R. Durrant, J. Am. Chem. Soc. 2015, 137, 6629; b) C. A. Mesa, L. Francas, K. R. Yang, P. Garrido-Barros, E. Pastor, Y. Ma, A. Kafizas, T. E. Rosser, M. T. Mayer, E. Reisner, M. Gratzel, V. S. Batista, J. R. Durrant, Nat. Chem. 2020, 12, 82; c) E. Pastor, F. Le Formal, M. T. Mayer, S. D. Tilley, L. Francas, C. A. Mesa, M. Gratzel, J. R. Durrant, Nat. Commun. 2017, 8, 14280. 
[12] a) A. M. Schimpf, C. E. Gunthardt, J. D. Rinehart, J. M. Mayer, D. R. Gamelin, J. Am. Chem. Soc. 2013, 135, 16569; b) S. Feldmann, S. Macpherson, S. P. Senanayak, M. Abdi-Jalebi, J. P. H. Rivett, G. J. Nan, G. D. Tainter, T. A. S. Doherty, K. Frohna, E. Ringe, R. H. Friend, H. Sirringhaus, M. Saliba, D. Beljonne, S. D. Stranks, F. Deschler, Nature Photonics 2020, 14, 123; c) K. E. Hughes, K. H. Hartstein, D. R. Gamelin, ACS Nano 2018, 12, 718; d) T. Liu, D. Xiang, Y. Zheng, Y. Wang, X. Wang, L. Wang, J. He, L. Liu, W. Chen, Adv. Mater. 2018, 30, 1804470.

[13] a) B. Tan, S. R. Raga, K. J. Rietwyk, J. Lu, S. O. Fürer, J. C. Griffith, Y.-B. Cheng, U. Bach, Nano Energy 2021, 82, 105658; b) S. Elmas, W. Beelders, J. Nash, T. J. Macdonald, M. Jasieniak, H. J. Griesser, T. Nann, RSC Adv. 2016, 6, 70691.

[14] T. Berger, D. Monllor-Satoca, M. Jankulovska, T. Lana-Villarreal, R. Gomez, Chemphyschem 2012, 13, 2824.

[15] C. A. Mesa, A. Kafizas, L. Francas, S. R. Pendlebury, E. Pastor, Y. Ma, F. Le Formal, M. T. Mayer, M. Gratzel, J. R. Durrant, J. Am. Chem. Soc. 2017, 139, 11537.

[16] B. D. Paulsen, K. Tybrandt, E. Stavrinidou, J. Rivnay, Nat. Mater. 2020, 19, 13.

[17] S. Selim, E. Pastor, M. Garcia-Tecedor, M. R. Morris, L. Francas, M. Sachs, B. Moss, S. Corby, C. A. Mesa, S. Gimenez, A. Kafizas, A. A. Bakulin, J. R. Durrant, J. Am. Chem. Soc. 2019, 141, 18791.

[18] a) M. Bledowski, L. Wang, A. Ramakrishnan, A. Betard, O. V. Khavryuchenko, R. Beranek, Chemphyschem 2012, 13, 3018; b) M. Bledowski, L. Wang, S. Neubert, D. Mitoraj, R. Beranek, J. Phys. Chem. C 2014, 118, 18951; c) L. Wang, D. Mitoraj, S. Turner, O. V. Khavryuchenko, T. Jacob, R. K. Hocking, R. Beranek, ACS Catal. 2017, 7, 4759; d) P. Longchin, D. Mitoraj, O. M. Reyes, C. Adler, N. Wetchakun, R. Beranek, J. Phys. Energy 2020, 2, 044001. 


\section{Supporting Information}

\section{Photodoping and Fast Charge Extraction in Ionic Carbon Nitride Photoanodes}

\section{Experimental}

\section{Materials}

Melamine (99\%) and $\mathrm{Na}_{2} \mathrm{SO}_{4}(\geq 99 \%)$ were provided by Sigma Aldrich, methanol ( $\left.\geq 99.9 \%\right), \mathrm{KOH}(99 \%)$, $\mathrm{NaOH}(99 \%)$ and $\mathrm{HCl}(37 \%)$ by Merck. Ethanol (99.8\%) was purchased by VWR and FTO Pilkington TEC 15 by Xop Fisica SL.

\section{Synthesis}

Synthesis of the PHI solution: The synthesis of PHI solution was carried out according to literature..$^{[1]}$ In brief, $0.2 \mathrm{~g}$ of $\mathrm{NaOH}, 0.56 \mathrm{~g}$ of $\mathrm{KOH}$ and $1.5 \mathrm{~g}$ of melamine were ground and heated in a lid-covered crucible for $2 \mathrm{~h}$ at $330{ }^{\circ} \mathrm{C}$ in a muffle furnace (heating rate: $5^{\circ} \mathrm{C} \mathrm{min}{ }^{-1}$ ). The obtained orange solid was dissolved in deionized water, whereby the insoluble parts were filtered with a paper filter. The solution was filtered with a $0.2 \mu \mathrm{m}$ PTFE syringe filter and filled into a cellulose membrane sack (pore size: 3.5 $\mathrm{kDa})$. After 5 days of dialysis against deionized water, the solution was concentrated by evaporation at $70{ }^{\circ} \mathrm{C}$ until a concentration of $3.5-4.5 \mathrm{mg} \mathrm{mL}^{-1}$ was obtained.

Preparation of the PHI-DB/S450 electrodes: The PHI-DB/S450 films were prepared following the methodology by Adler et al. ${ }^{[2]}$ Briefly, $12.5 \mathrm{~mL}$ of the $\mathrm{PHI}$ solution was gelled with $32.5 \mathrm{~mL}$ of ethanol and, after centrifugation and washing (three times with ethanol), the gel was liquified by vortexing. 200 $\mu \mathrm{L}$ of the gel were used to produce the films on FTO glasses by the doctor blading technique. After drying the electrodes in air at room temperature, the samples were calcined at $450{ }^{\circ} \mathrm{C}$ under $\mathrm{N}_{2}$ flow in a tube furnace (heating rate: $5^{\circ} \mathrm{C} \min ^{-1}$ ).

Preparation of the PHI-DB/H electrodes: The PHI-DB/H400 films were prepared following the methodology by Adler et al. ${ }^{[2]}$ Briefly, $25 \mathrm{~mL}$ of $\mathrm{PHI}$ solution was gelled with $0.5 \mathrm{~mL}$ of $37 \% \mathrm{HCl}$ and centrifuged. The gel was liquified by vortexing and $200 \mu \mathrm{L}$ of the gel were used to produce the films on FTO glasses by the doctor blading technique. After drying the electrodes in air at room temperature, the samples were calcined at $400{ }^{\circ} \mathrm{C}$ under $\mathrm{N}_{2}$ flow in a tube furnace (heating rate: $5^{\circ} \mathrm{C} \min ^{-1}$ ).

Preparation of $\mathrm{BiVO}_{4}$ electrode: The bismuth vanadate electrode was prepared according to previous studies. $^{[3]}$ 


\section{Photolectrochemical measurements:}

Photoelectrochemical measurements in Figure 1a, Figure S5 and Figure S7 were performed with 2 sun irradiation (AM 1.5G) in $0.1 \mathrm{M} \mathrm{Na}_{2} \mathrm{SO}_{4}$ with $10 \mathrm{v} \% \mathrm{MeOH}$ electrolyte at $\mathrm{pH} 7.0$ using a SP-300 Biologic potentiostat with a three-electrode setup using a Pt counter electrode, a $\mathrm{Ag} / \mathrm{AgCl}(3.5 \mathrm{M} \mathrm{KCl})$ reference electrode and the PHI-coated FTO electrode as working electrode. The electrodes were irradiated from backside with an Ushio $150 \mathrm{~W}$ Xe lamp in a light-condensing lamp housing (LOT-Oriel GmbH). LSV measurements (Figure 1a, Figure S5) were performed with a scan rate of $5 \mathrm{mV} \mathrm{s}^{-1}$. Impedance measurements were carried out in a frequency range of $100 \mathrm{kHz}$ to $100 \mathrm{mHz}$ in dark or light at $0.7 \mathrm{~V}$ vs. RHE (Figure S7).

The photoreforming of methanol was performed in a two-electrode setup without applied bias using a $\mathrm{Pt}$ counter electrode and the PHI-coated FTO electrode as working electrode separated with a Nafionimpregnated glass frit. A mixture of $0.1 \mathrm{M} \mathrm{Na}_{2} \mathrm{SO}_{4}$ and $10 \mathrm{v} \% \mathrm{MeOH}(\mathrm{pH} 2.0)$ served as electrolyte in the anodic compartment (PHI-electrode) while pure $0.1 \mathrm{M} \mathrm{Na}_{2} \mathrm{SO}_{4}(\mathrm{pH} 2.0)$ was used in the cathodic one (Pt electrode). The cathodic compartment was closed with a rubber septum and both compartments were purged 30 min with $\mathrm{Ar}$ before the measurements. The electrode was irradiated from back side under one sun illumination. Samples of the head space $(50 \mu \mathrm{L})$ of the cathodic compartment were taken every $30 \mathrm{~min}$ and were analyzed with a Shimadzu GC-2010 Plus equipped with a BID detector and a ShinCarbon ST column. The discharge He gas flow was $70 \mathrm{~mL} \mathrm{~min}^{-1}$ and the column temperature was $35^{\circ} \mathrm{C}$ for $2.5 \mathrm{~min}$ followed by $20^{\circ} \mathrm{C} \mathrm{min}-1$ heating up to $180^{\circ} \mathrm{C}$ for $0.5 \mathrm{~min}$. The peak area of hydrogen as indicator for hydrogen production was taken for the final plot.

If not denoted otherwise, the PHI-DB/S450 photoanodes were activated prior the measurements with cyclic voltammetry (2 cycles) by applying potentials from 1.6 to $0 \mathrm{~V}$ vs. RHE with a scan rate of $20 \mathrm{mV}$ $\mathrm{s}^{-1}$ in $0.1 \mathrm{M} \mathrm{Na}_{2} \mathrm{SO}_{4}$ at $\mathrm{pH} 7.0$ under $10 \mathrm{~mW} \mathrm{~cm}^{-2} 365 \mathrm{~nm}$ LED (if measurement afterwards was performed with LED) or 2 sun (if measurement was performed with Xe-lamp afterwards) irradiation.

\section{Optical measurements:}

Photoinduced absorption spectroscopy (PIA) and corresponding transient photocurrent: PIA and transient photocurrent measurements were performed with a $365 \mathrm{~nm}$ LED. The intensity of the LED was adjusted by varying the applied currents using a DC power supply (TTi QL564P). A liquid light guide is used to direct the LED output to the sample. Pulsed LED illumination was generated using a MOSFET transistor, where the gate is modulated by a DAQ card (National Instruments, USB-6361), where the $\mathrm{DAQ}$ card switches the gate with a voltage signal which initiates a light pulse, whilst simultaneously triggering data acquisition. Excitation densities were measured using a silicon photodiode (Thorlabs S120UV) fitted with a digital power meter (Thorlabs PM100). A 100 W Bentham IL1 tungsten lamp coupled to a monochromator (OBB-2001, Photon Technology International) was used as the probe light. The system and data acquisition were controlled by a home-programmed Labview software.

Unless stated otherwise, the measurements were performed with $5 \mathrm{~mW} \mathrm{~cm}^{-2}$. During the measurements, the sample was irradiated through the backside. The LED was on for $5 \mathrm{~s}$ and off for 45 $\mathrm{s}$, where the transient photocurrents and the optical signals (recorded as $\mathrm{m} \triangle \mathrm{OD}$, the change in optical 
density/1000) were measured for the duration of the $50 \mathrm{~s}$ in total. If the samples were treated with pre-

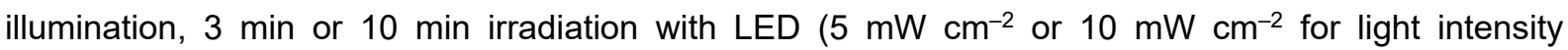
dependency measurement in Figure S3) was carried out directly before the PIA/transient photocurrent measurements (Figure S1, Figure 1b, Figure 2a, b). After pre-illumination experiments, the sample was kept in dark for at least 15 min before using it for the next experiment. During this time, the optical signal was observed to recover to the values obtained prior to pre-illumination. If potential was applied, a threeelectrode setup was used with an Autolab potentiostat (PGSTAT 101, Metrohm) using a Pt mesh and a $\mathrm{Ag} / \mathrm{AgCl}$ (saturated $\mathrm{KCl}$ ) as counter and reference electrode, respectively.

Nanosecond transient absorption (ns-TA): The PHI-DB/S450 films were not activated prior the ns-TA measurements. The ns-TA spectra were performed upon $355 \mathrm{~nm}$ pump pulses produced by a continuum surelite Nd:YAG laser system (pulse duration $5 \mathrm{~ns}$, repetition rate $10 \mathrm{~Hz}$ ). The probe light is provided by a $75 \mathrm{~W}$ xenon arc lamp. Spherical concave mirrors are used to focus the probe beam into the samples and then to send the beam to the monochromator (Acton, Princeton Instruments). The spectrally selected probe light is detected by a Hamamatsu R928 photomultiplier. $355 \mathrm{~nm}$ band-stop filter was used to eliminate the pump scattering and a short-pass filter $(520 \mathrm{~nm})$ was used to get rid of the red component in the excitation pump.

Transient photocurrent (microsecond - second): TPC measurements were carried out with a $355 \mathrm{~nm}$ Nd:YAG laser (OPOTEK Inc., Opolette 355 laser system, 7 ns pulse width) with a frequency of $0.8 \mathrm{~Hz}$ and an intensity of $300 \mu \mathrm{J} \mathrm{cm}^{-2}$. In order to apply potential, a three-electrode setup was used with a Autolab potentiostat (PGSTAT 101, Metrohm) using a Pt mesh and a $\mathrm{Ag} / \mathrm{AgCl}$ (saturated $\mathrm{KCl}$ ) as counter and reference electrode, respectively. The obtained data were processed through an amplifier (Costronics), where the microsecond - millisecond data were recorded by an oscilloscope (Tektronics TDS 2012B), and the millisecond to second data were recorded with a DAQ card (National Instruments, NI USB-6361). The system and data acquisition were controlled by a home programmed Labview software.

Calculation of photon flux:

365 nm LED for PIA:

$$
\begin{aligned}
& \frac{\text { Number of photons }}{\text { time }}=\frac{\text { power density }}{\frac{\mathrm{h}}{\mathrm{c} \cdot \lambda}}=9.2 \cdot 10^{15} \frac{\mathrm{J}}{\mathrm{s} \cdot \mathrm{cm}^{2}} \\
& \frac{\text { Number of photons }}{\text { time }}=\frac{\frac{\mathrm{h}}{\mathrm{c} \cdot \lambda}}{\text { laser pulse width }}=7.7 \cdot 10^{22} \frac{\mathrm{J}}{\mathrm{s} \cdot \mathrm{cm}^{2}}
\end{aligned}
$$

With $\mathrm{h}$ and $\mathrm{c}$ as the Planck constant and speed of light, respectively. The power density of the LED is 5 $\mathrm{mW} \mathrm{cm}{ }^{-2}$ with a wavelength $(\lambda)$ of $365 \mathrm{~nm}$. The excitation density, $\lambda$ and laser pulse width of the laser is $300 \mu \mathrm{J} \mathrm{cm}^{-2}, 355 \mathrm{~nm}$ and $7 \mathrm{~ns}$, respectively. 


\section{Results and Discussion}

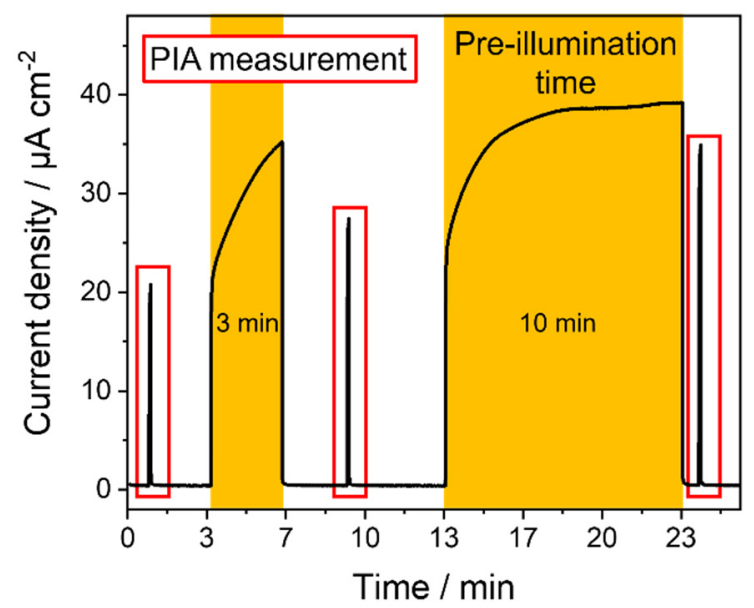

Figure S1. Chronoamperometry of PHI-DB/S450 at $1.1 \mathrm{~V}$ vs. RHE in $0.1 \mathrm{M} \mathrm{Na}_{2} \mathrm{SO}_{4}+10 \mathrm{v} \% \mathrm{MeOH}$ for a PIA / transient photocurrent measurement to investigate the effect of pre-illumination. PIA / transient photocurrent measurements are labelled in red and pre-illumination of $3 \mathrm{~min}$ or $10 \mathrm{~min}$ is shown in yellow. Excitation: $365 \mathrm{~nm}$ LED $\left(5 \mathrm{~mW} \mathrm{~cm}^{-2}\right)$.
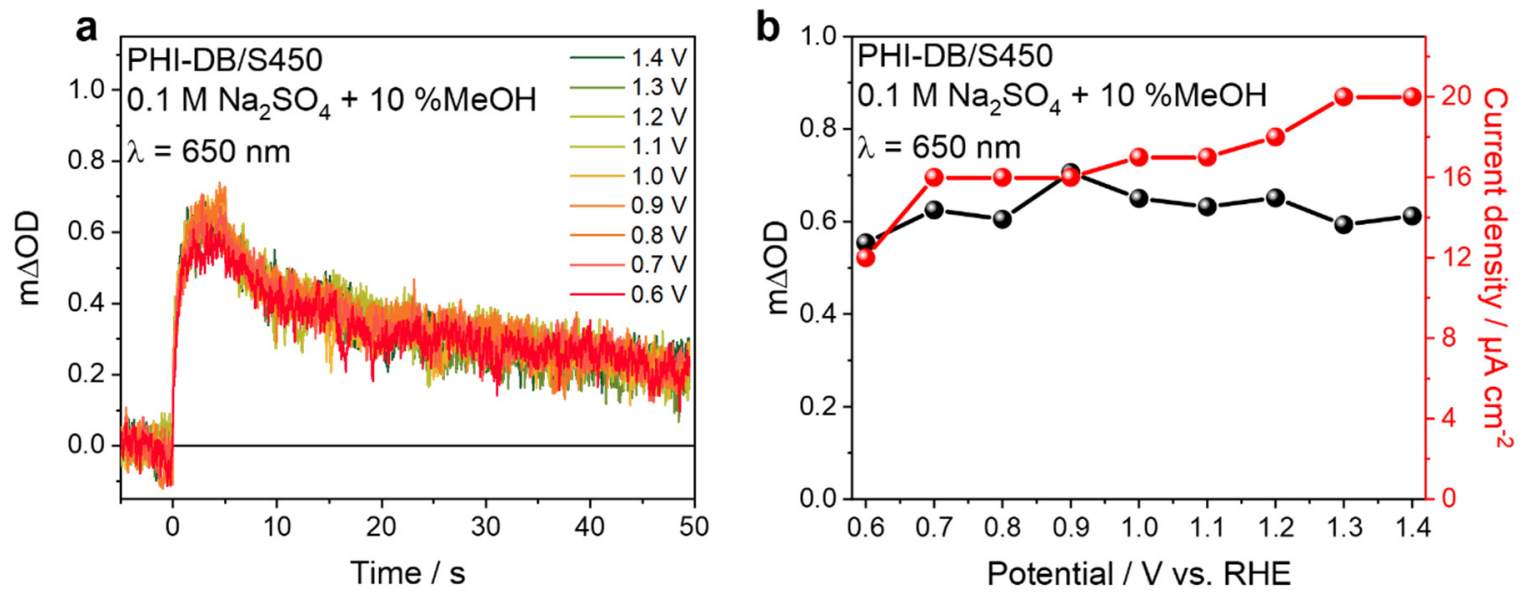

Figure S2. a) PIA of PHI-DB/S450 without pre-illumination at potentials from $0.6 \mathrm{~V}$ to $1.4 \mathrm{~V}$ vs. RHE in $0.1 \mathrm{M} \mathrm{Na}_{2} \mathrm{SO}_{4}$ $+10 \mathrm{v} \% \mathrm{MeOH}$ under $365 \mathrm{~nm}$ LED illumination $\left(5 \mathrm{~mW} \mathrm{~cm}^{-2}\right)$ for $5 \mathrm{~s}$ at $650 \mathrm{~nm}$ and b) $\mathrm{m} \Delta \mathrm{OD}$ values (black) and current density (red) after $5 \mathrm{~s}$ of illumination. 

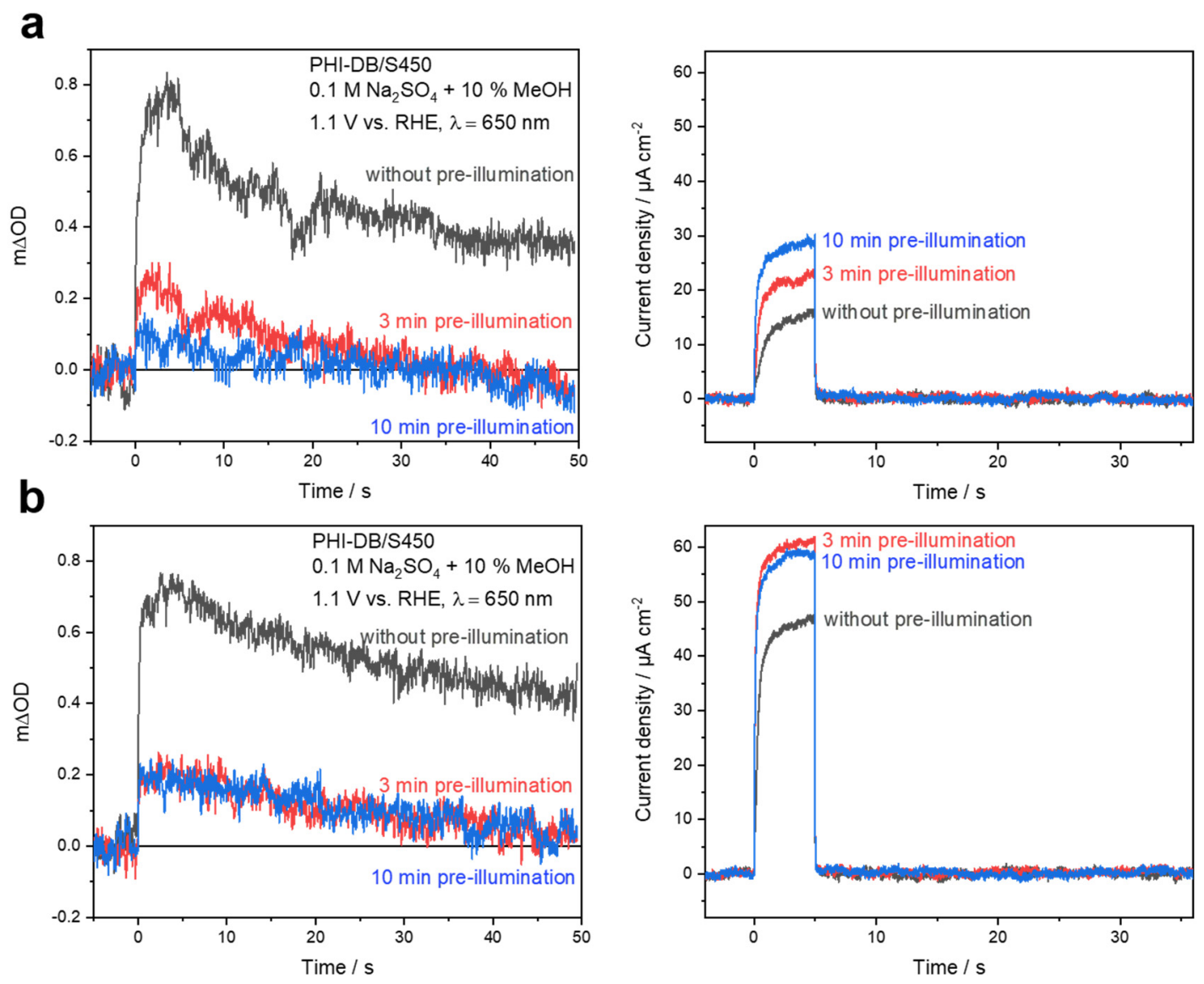

Figure S3. PIA of PHI-DB/S450 without and with 3 or 10 min pre-illumination and corresponding transient photocurrent at $1.1 \mathrm{~V}$ vs. RHE in $0.1 \mathrm{M} \mathrm{Na}_{2} \mathrm{SO}_{4}+10 \mathrm{v} \% \mathrm{MeOH}$ under a) $5 \mathrm{~mW} \mathrm{~cm}^{-2}$ and b) $10 \mathrm{~mW} \mathrm{~cm}^{-2}$ with 365 $\mathrm{nm}$ LED illumination for $5 \mathrm{~s}$. Optical signals in $a$ and $\mathrm{b}$ were probed at $650 \mathrm{~nm}$.

\section{Note S1. Nanosecond-transient absorption spectroscopy of PHI-DB/S450}

We apply nanosecond-transient absorption (ns-TA) spectroscopy under bias-free conditions to investigate the charge kinetics of the PHI-DB/S450 films on a timescale of $50 \mathrm{~ns}$ to $5 \mu \mathrm{s}$. We perform the spectroscopic characterization (see Figure $\mathrm{S} 4$ ) under various experimental conditions, i.e., studying the films in air, neat water and in water upon addition of methanol.

The transient absorption spectra at a delay time of 100 ns (see Figure S4a) reveal a pronounced negative feature (370-500 nm), due to both ground-state bleach and strong emission, accompanied by a broad band in the visible $(500-800 \mathrm{~nm})$. The latter is attributed to the absorption of photogenerated electrons. At even longer delay times (see Figure S4b) the negative differential absorption feature is superimposed by a (strong) photoinduced absorption (PIA) feature at $400 \mathrm{~nm}$ rendering the net $\Delta \mathrm{OD}$ slightly positive in the region below $500 \mathrm{~nm}$.

By subtracting the emission contribution from the PIA spectra, it becomes apparent that both PIA bands, i.e., at $400 \mathrm{~nm}$ and above $520 \mathrm{~nm}$, show similar decay kinetics in the temporal range between $50 \mathrm{~ns}$ and 5 us when the sample is exposed to air (Figure S4c). The corresponding decay kinetics are 
characterized by a half-live time of $t_{50 \%} \approx 1.6 \mu$ s suggesting that both PIA bands result from the same photogenerated species or the decay of photogenerated species is dominated by similar processes.

Addition of $\mathrm{MeOH}$ to water as additional hole scavenger of PHI-DB/S450 (see Figure S4d) accelerated the decay kinetics of the PIA signal recorded between 500 and $800 \mathrm{~nm}$ in the $\mathrm{MeOH} /$ water mixture with $t_{50 \%}$ shortened to $0.7 \mu \mathrm{s}$. Under the assumption that the reaction rate of oxygen reaction with the trapped electrons remains constant, the shortened $t_{50 \%}$ indicates a faster recombination in the presence of the hole quencher $\mathrm{MeOH}$. This observation is in line with our previous studies on ionic-type PCN suspensions ${ }^{[4]}$ and the accelerated decay can be interpreted in terms of a trapping/detrapping mediated bimolecular (band-to-band-like) recombination that becomes faster when more electrons accumulate in the material during the optical experiment in the presence of an effective hole quencher. ${ }^{[4 a]}$ Keeping the pump intensity constant, the addition of methanol (10 vol\%) makes the decay of the photogenerated electrons to follow pseudo-first-order kinetics, which does not change upon further increase (up to 30 vol\%) of methanol concentration (Figure S4e). As expected, the decay kinetics tend to become independent of electron concentration when the electron concentration becomes much larger due to hole scavenging by methanol compared to the concentration of photogenerated holes.

In general, the results from ns-TA spectroscopy under bias-free conditions show that our PHI photoanodes behave similarly to the ionic (PHI-type) PCN powder materials that have been previously studied in form of suspensions, ${ }^{[4]}$ and which are known to store photogenerated electrons in form of $\mathrm{PHI}^{-}$anion radicals when $\mathrm{MeOH}$ is added as an electron donor. ${ }^{[4 b, 5]}$ This further emphasizes that the nature and key characteristics of ionic $\mathrm{PHI}$ are preserved in our $\mathrm{PHI}$ photoanodes.
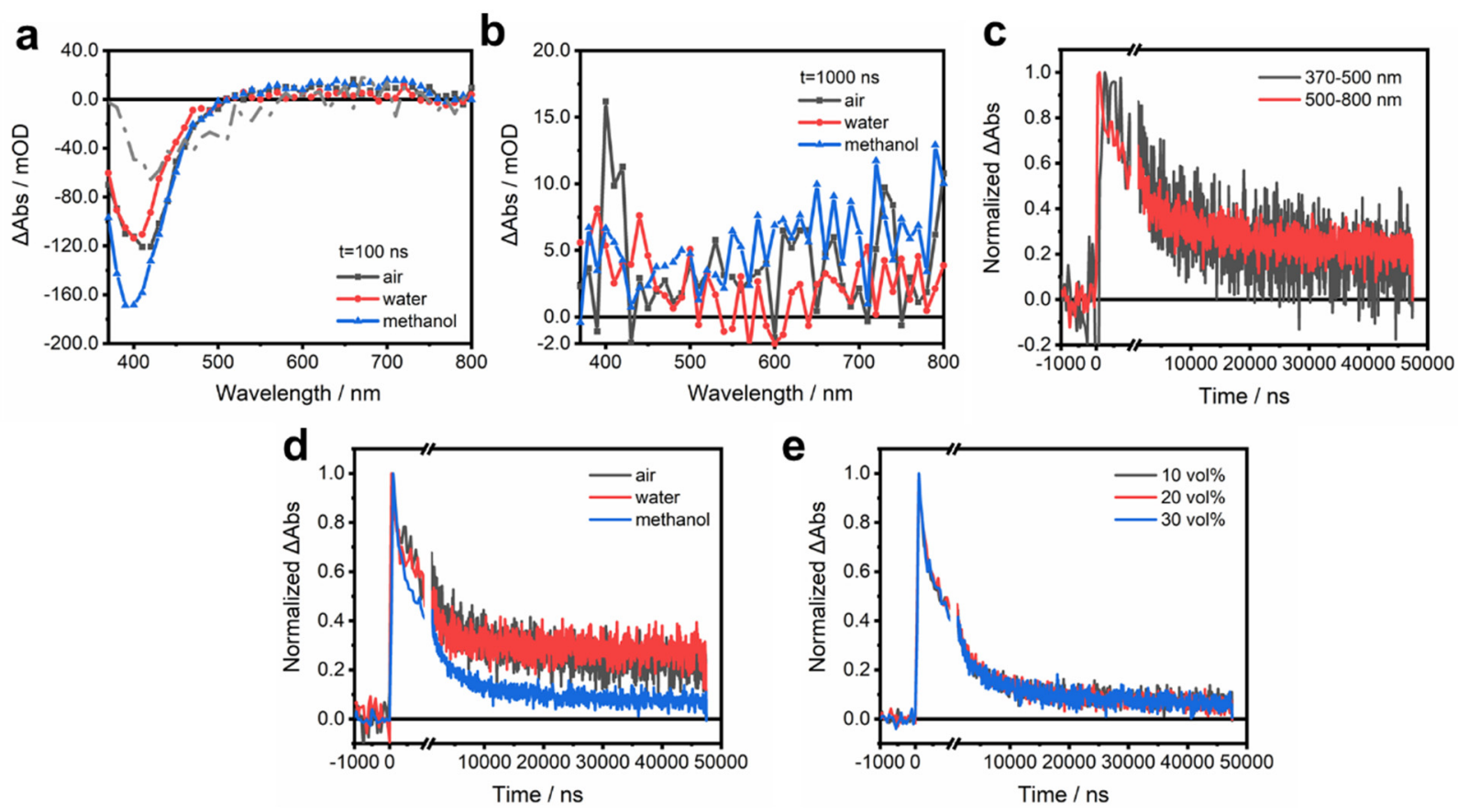

Figure S4. Comparison of ns-TA spectra of PHI-DB/S450 films in air, water and methanol/water mixture (10 vol\%) after $100 \mathrm{~ns}(\mathrm{a})$ and $1000 \mathrm{~ns}$ (b) upon $355 \mathrm{~nm}$ excitation $\left(\sim 10 \mathrm{~mJ} / \mathrm{cm}^{2}\right)$. The grey dashed line represents the shape of the corresponding inverted ns-emission spectrum at $30 \mathrm{~ns}$ in a methanol/water mixture (10 vol\%) scaled to fit the respective figure. c) Normalized decay kinetics of PIA signal in air from $370-500 \mathrm{~nm}$ and $500-800 \mathrm{~nm}$ respectively. d) The normalized decay kinetics of PIA signal from $500-800 \mathrm{~nm}$. e) PIA of PHI-DB/S450 in various volume ratios of methanol within the wavelength range of 500 to $800 \mathrm{~nm}$ under $355 \mathrm{~nm}$ excitation. 
a

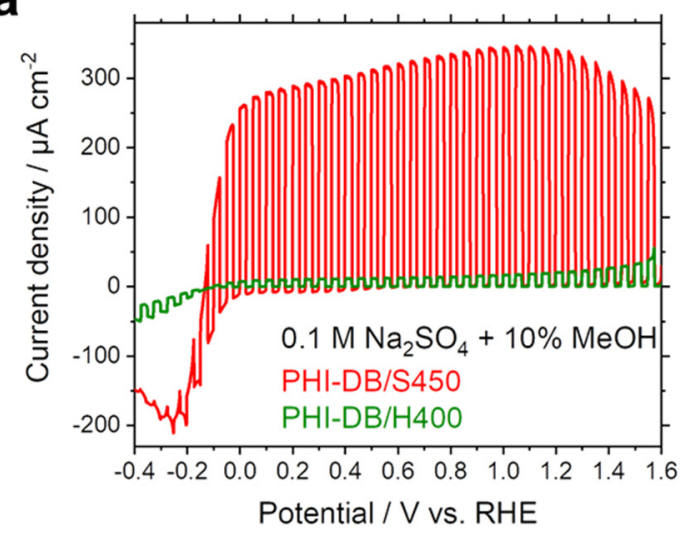

b

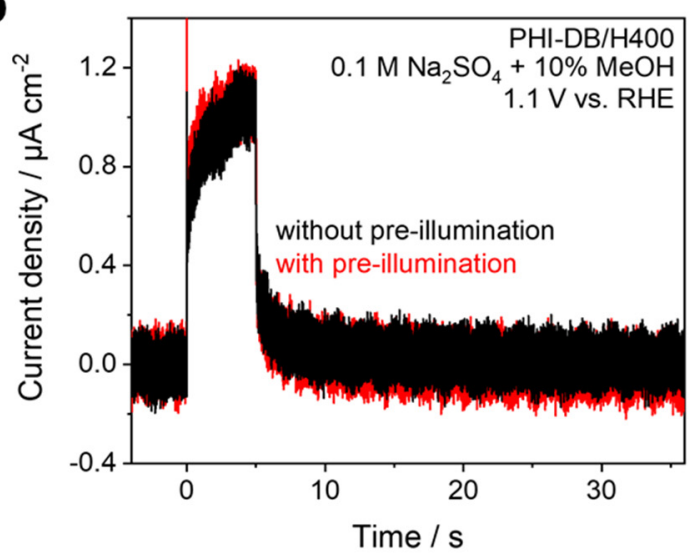

Figure S5. Comparison with the dense PHI-DB/H400 film: a) LSV curves (scanned in cathodic direction with a sweep-rate of $5 \mathrm{mV} \mathrm{s}^{-1}$ ) of porous PHI-DB/S450 and dense PHI-DB/H400 in $0.1 \mathrm{M} \mathrm{Na}_{2} \mathrm{SO}_{4}$ with $10 \mathrm{v} \%$ methanol (pH 7.0) upon on/off illumination (2 sun, $5 \mathrm{~s}$ light/ $5 \mathrm{~s}$ dark) from back side. b) Photocurrent transients measured for the dense PHI-DB/H400 film in $0.1 \mathrm{M} \mathrm{Na} 2 \mathrm{SO} 4+10 \mathrm{v} \%$ methanol at $1.1 \mathrm{~V}$ vs. RHE without (black) and with $10 \mathrm{~min}$ (red) pre-illumination directly before measurement. Excitation with $365 \mathrm{~nm}$ LED $(5 \mathrm{~mW} \mathrm{~cm}-2)$ for $5 \mathrm{~s}$ and dark for $45 \mathrm{~s}$.

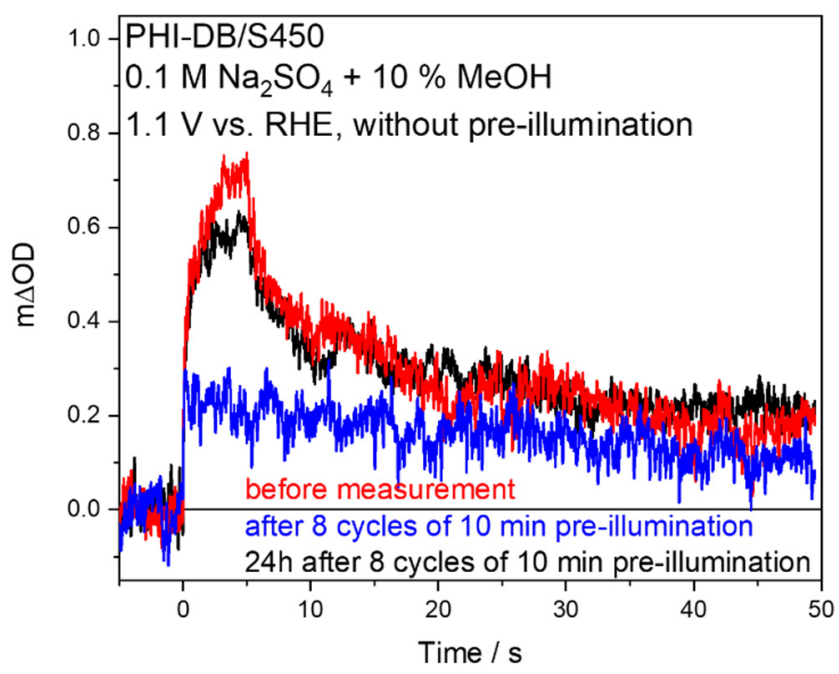

Figure S6. PIA of PHI-DB/S450 before measurement, after treatment of 8 cycles of 10 min pre-illumination and 24h after this treatment at $1.1 \mathrm{~V}$ vs. RHE in $0.1 \mathrm{M} \mathrm{Na}_{2} \mathrm{SO}_{4}+10 \mathrm{v} \% \mathrm{MeOH}$ under $5 \mathrm{~mW} \mathrm{~cm}^{-2} 365 \mathrm{~nm}$ LED illumination for $5 \mathrm{~s}$, probed at $650 \mathrm{~nm}$. 
a

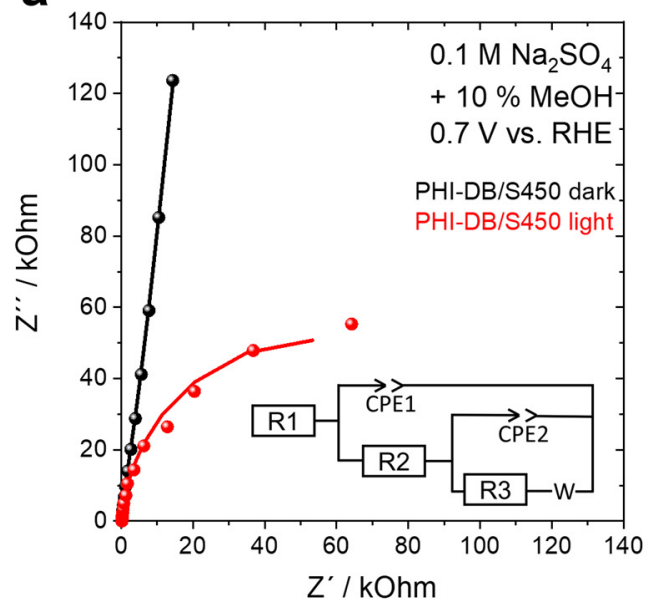

b

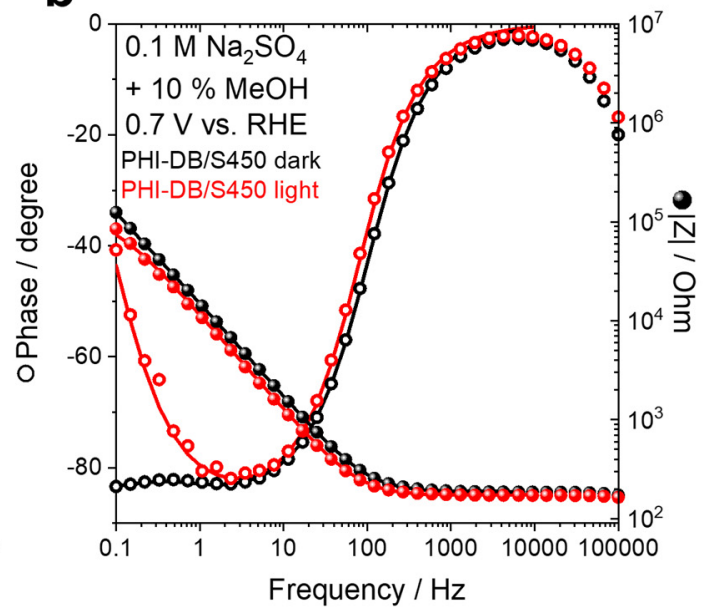

Figure S7. a) Nyquist impedance plot and b) Bode impedance plot of PHI-DB/S450 at $0.7 \mathrm{~V}$ vs. RHE in $0.1 \mathrm{M}$ $\mathrm{Na}_{2} \mathrm{SO}_{4}+10 \mathrm{v} \% \mathrm{MeOH}$ without and with light illumination (2 sun, AM1.5G filter). Dots: experimental data, line: fit obtained with equivalent circuit shown in the inset in a) with R2 values (corresponding to the film resistance) in dark of $4 \cdot 10^{5} \Omega$ and light of $1 \cdot 10^{-12} \Omega$.
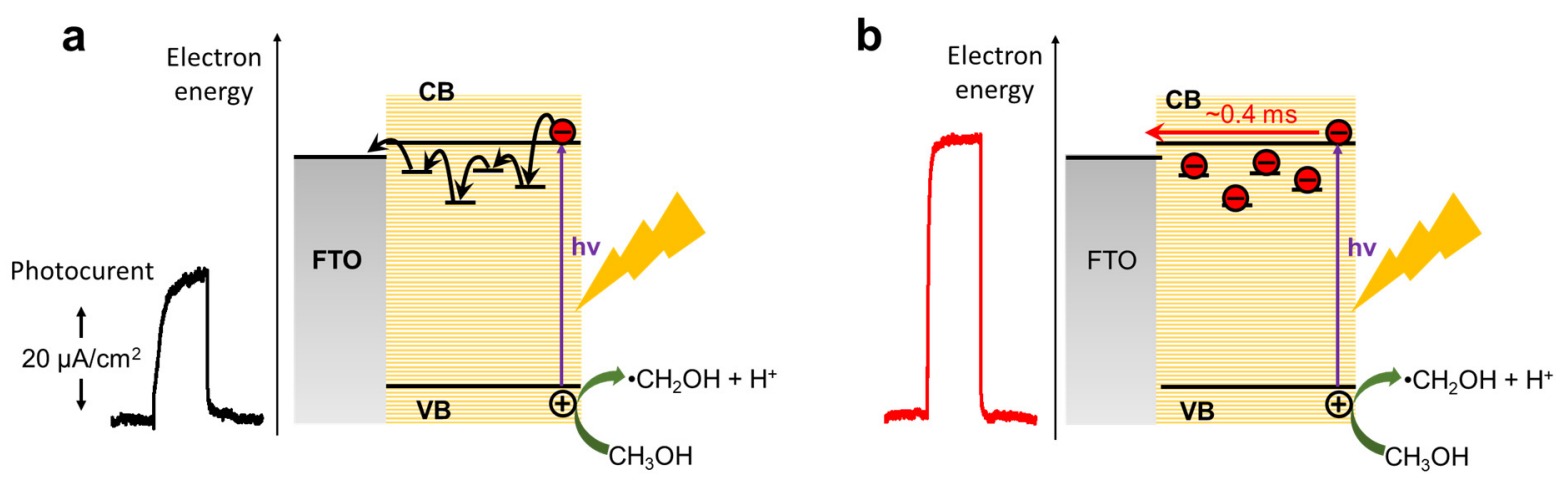

Figure S8. A simplified scheme showing the effect of photodoping on the electron transport through the PHI film. (a) In the absence of photodoping, a significant proportion of photogenerated electrons undergo deep charge trapping, impeding the efficiency and kinetics of electron extraction to the external circuit; (b) In the presence of photodoping resulting in the filling / passivation of trap states, electron extraction becomes more efficient. All recombination pathways are omitted for the sake of clarity. 


\section{a}

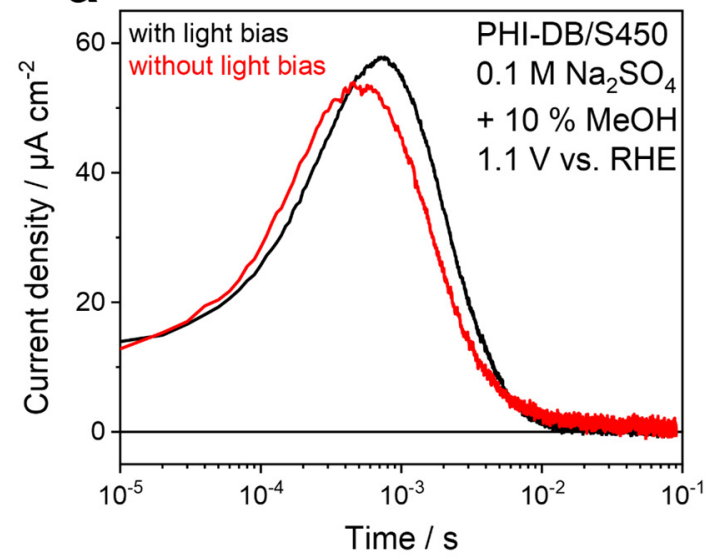

b

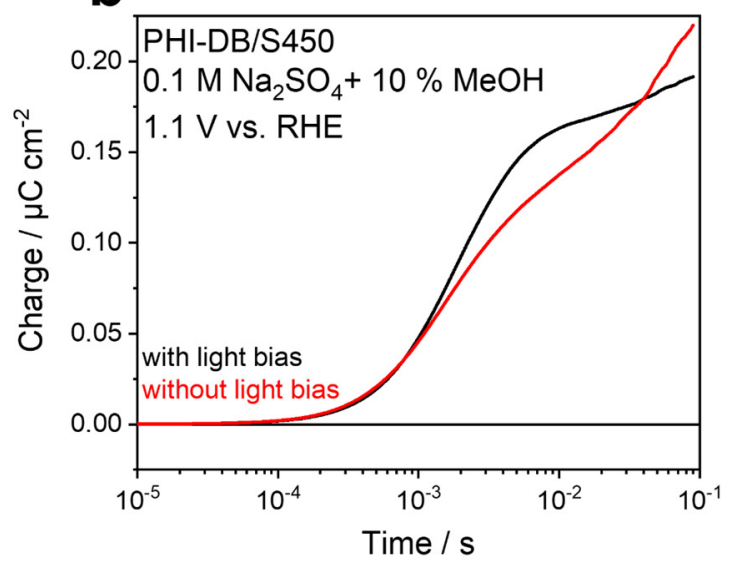

Figure S9. a) TPC and b) total charge without and with background light bias from back side $\left(5 \mathrm{~mW} \mathrm{~cm}^{-2}, 365 \mathrm{~nm}\right.$ LED) for PHI-DB/S450 in $0.1 \mathrm{M} \mathrm{Na}_{2} \mathrm{SO}_{4}+10 \mathrm{v} \% \mathrm{MeOH}$ and excitation with $355 \mathrm{~nm}$ pulsed laser.

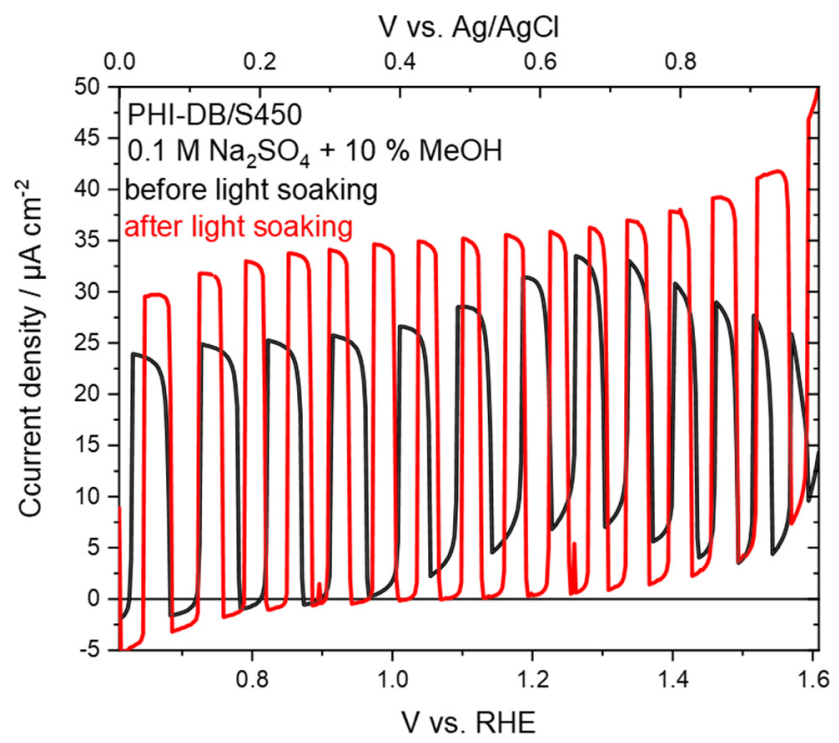

Figure S10. LSV of PHI-DB/S450 before and after TPC measurements with background light bias $\left(5 \mathrm{~mW} \mathrm{~cm}^{-2}, 365\right.$ $\mathrm{nm}$ LED) in $0.1 \mathrm{M} \mathrm{Na}_{2} \mathrm{SO}_{4}+10 \mathrm{v} \% \mathrm{MeOH}$. 

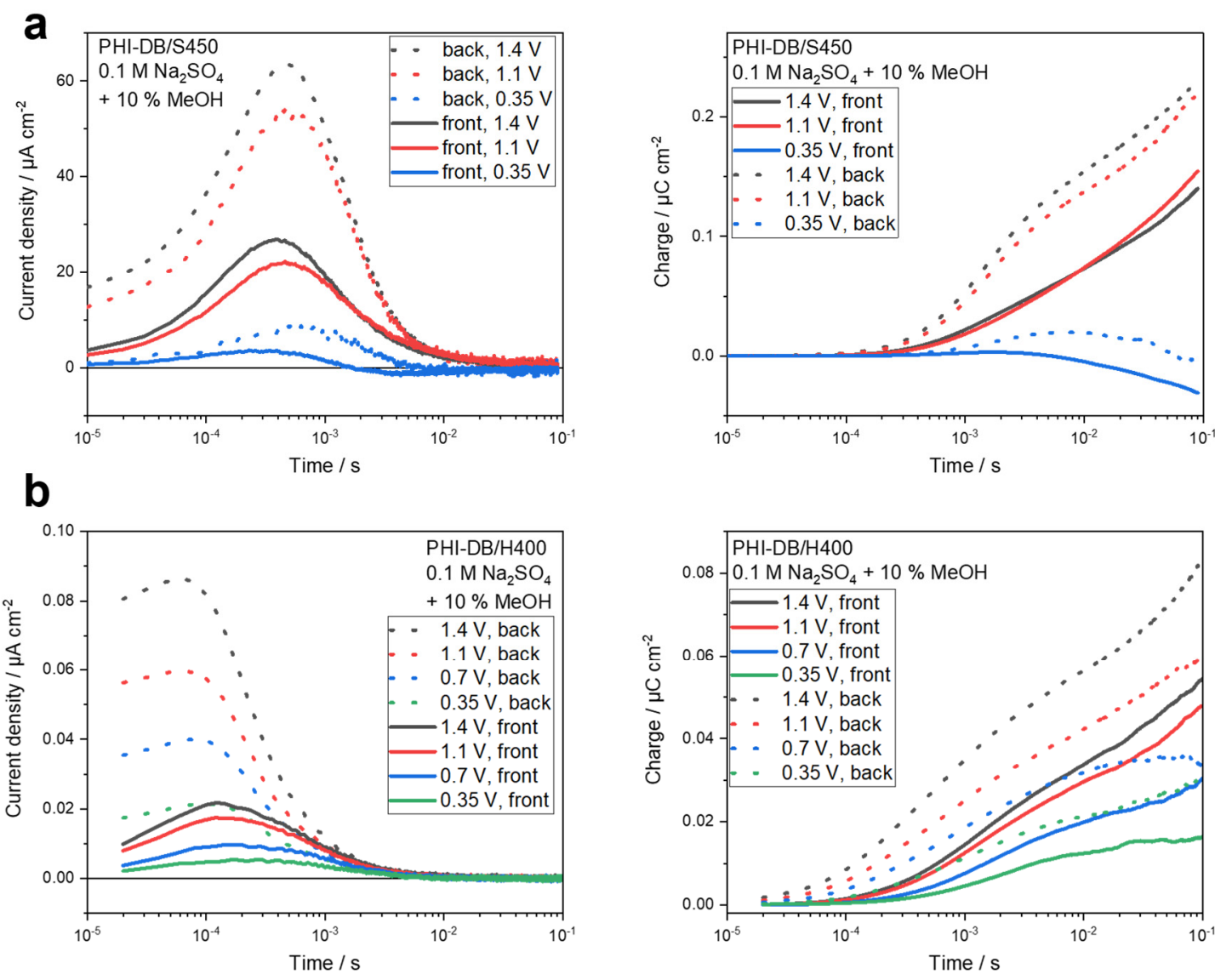

Figure S11. TPC for front and back side irradiation for a) PHI-DB/S450 and b) PHI-DB/H400 and corresponding total charge in $0.1 \mathrm{M} \mathrm{Na}_{2} \mathrm{SO}_{4}+10 \mathrm{v} \% \mathrm{MeOH}$ and excitation with $355 \mathrm{~nm}$ pulsed laser.

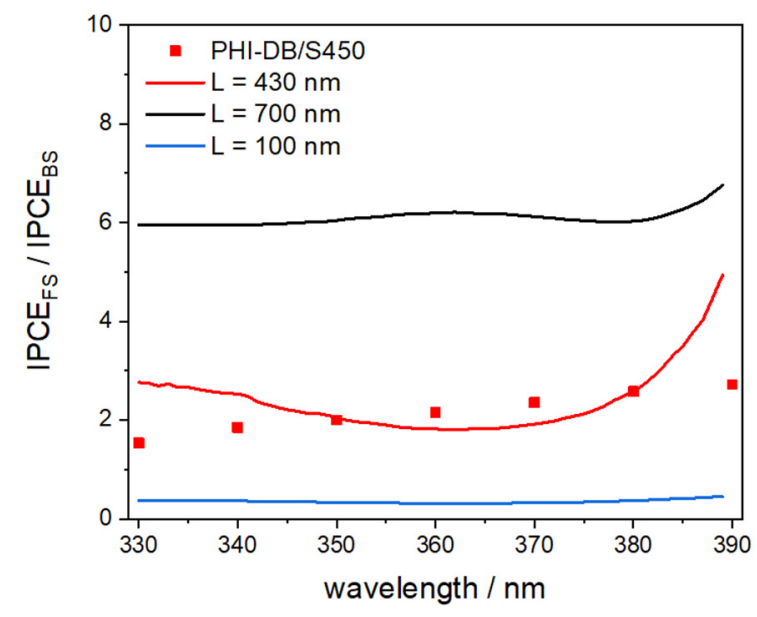

Figure S12. Experimental ratio of the IPCE values ${ }^{[2]}$ recorded under the frontside (FS) and the backside (BS) illumination (red points). Theoretical traces (solid lines) for different electron diffusion lengths $L$ as determined using the model of Soedergren et al. ${ }^{[6]}$ The ratio of IPCE ${ }_{\mathrm{FS}} / \mathrm{IPCE} \mathrm{BS}_{\mathrm{BS}}$ was calculated as $-\left(1-R_{F S}\right)\left[(L \alpha+1) e^{2 d / L}-\right.$ $\left.2 L \alpha e^{\alpha d+d / L}+L \alpha-1\right] /\left(1-R_{B S}\right)\left[(L \alpha-1) e^{\alpha d+2 d / L}+(L \alpha+1) e^{\alpha d}-2 L \alpha e^{d / L}\right],,^{[7]}$ where $d$ is the film thickness of 400 $\mathrm{nm},{ }^{[2]} \alpha$ is the absorption coefficient ${ }^{[2]}$ and $R_{F S}$ and $R_{B S}$ are the reflectances measured from the electrolyte side (here neglected and taken as zero) and the substrate side, respectively. 


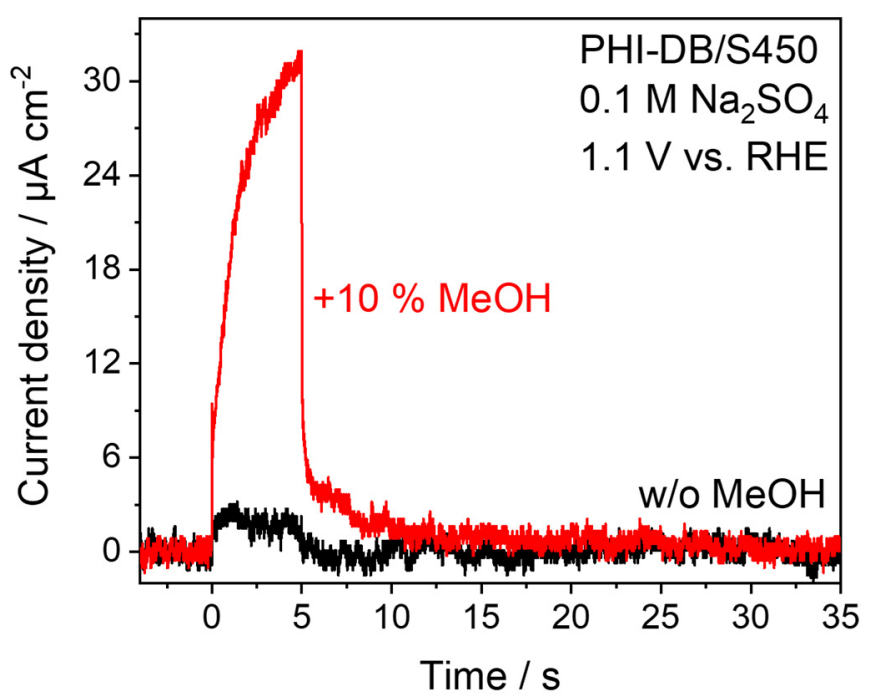

Figure S13. Pulsed LED photocurrent transients of PHI-DB/S450 in $0.1 \mathrm{M} \mathrm{Na}_{2} \mathrm{SO}_{4}$ with and without $10 \mathrm{v} \% \mathrm{MeOH}$ at $1.1 \mathrm{~V}$ vs. RHE without pre-illumination before the measurement. Excitation with $365 \mathrm{~nm} \mathrm{LED}\left(5 \mathrm{~mW} \mathrm{~cm}^{-2}\right)$ for 5 $\mathrm{s}$ and dark for 45. 


\section{References}

[1] I. Krivtsov, D. Mitoraj, C. Adler, M. Ilkaeva, M. Sardo, L. Mafra, C. Neumann, A. Turchanin, C. Li, B. Dietzek, R. Leiter, J. Biskupek, U. Kaiser, C. Im, B. Kirchhoff, T. Jacob, R. Beranek, Angew. Chem. Int. Ed. 2020, 59, 487.

[2] C. Adler, I. Krivtsov, D. Mitoraj, L. dos Santos-Gómez, S. García-Granda, C. Neumann, J. Kund, C. Kranz, B. Mizaikoff, A. Turchanin, R. Beranek, ChemSusChem 2021, 14, 2170.

[3] S. Selim, E. Pastor, M. Garcia-Tecedor, M. R. Morris, L. Francas, M. Sachs, B. Moss, S. Corby, C. A. Mesa, S. Gimenez, A. Kafizas, A. A. Bakulin, J. R. Durrant, J. Am. Chem. Soc. 2019, 141, 18791.

[4] a) W. Yang, R. Godin, H. Kasap, B. Moss, Y. Dong, S. A. J. Hillman, L. Steier, E. Reisner, J. R. Durrant, J. Am. Chem. Soc. 2019, 141, 11219; b) C. Li, E. Hofmeister, I. Krivtsov, D. Mitoraj, C. Adler, R. Beranek, B. Dietzek, ChemSusChem 2021, 14, 1728.

[5] a) A. Savateev, B. Kurpil, A. Mishchenko, G. Zhang, M. Antonietti, Chem. Sci. 2018, 9, 3584; b) A. Savateev, M. Antonietti, ChemCatChem 2019, 11, 6166; c) F. Podjaski, B. V. Lotsch, Adv. Energy Mater. 2021, 11, 2003049.

[6] S. Soedergren, A. Hagfeldt, J. Olsson, S.-E. Lindquist, J. Phys. Chem. 1994, 98, 5552.

[7] W. H. Leng, P. R. F. Barnes, M. Juozapavicius, B. C. O’Regan, J. R. Durrant, J. Phys. Chem. Lett. 2010, 1, 967. 